\title{
Coach-Auswahl durch Unternehmen - ritualisierte Selbstberuhigung im Closed Shop?
}

\author{
Louisa Schilling ${ }^{1}$ (D) $\cdot$ Thomas Webers $^{1}$ \\ Online publiziert: 22. Juli 2020 \\ (c) Der/die Autor(en) 2020
}

\section{Zusammenfassung}

Die gestiegene Nachfrage nach Coaching als Personalentwicklungsmaßnahme in den letzten Jahren resultiert in einem höheren Bedarf an kompetenten Coaches, die von den Unternehmen zuvor ausgewählt werden müssen. Darüber, wie Unternehmen die passenden Coaches auswählen und welche Anforderungen sie dabei zugrunde legen, existiert bislang nur wenig Forschung. Ersten Erkenntnissen zu Folge werden aus Ermangelung an belastbaren Anforderungskriterien lediglich Qualitätssurrogate überprüft. Die Intransparenz des Marktes sowie die fehlende Überprüfbarkeit von Zertifikaten und Ausbildungen sind dabei zwei mögliche Ursachen. Der vorliegende Artikel widmet sich dieser Frage und illustriert anhand einer empirischen qualitativen Interviewstudie die strategischen Hintergründe, Motive und Vorgehensweisen aller beteiligten Stakeholder in der Auswahl von Coaches. Dabei werden Coaches, Unternehmensvertreter und Dienstleistungsanbieter, welche die Coach-Auswahl stellvertretend übernehmen, befragt und ihre Aussagen für ein globales Bild des Markts gegenübergestellt. In der Betrachtung der Ergebnisse zeigt sich, dass die Auswahl von Coaches weiterhin ein Empfehlungsgeschäft und damit ein closed shop ist. Die wenig standardisierten Auswahlgespräche sowie die Vorherrschaft von Kompetenzvermutungen und Bauchgefühl als Auswahlkriterien lässt darauf schließen, dass die Anforderungen der Eignungsdiagnostik wenig erfüllt werden. Für die Verbesserung der Auswahlprozesse und eine stärkere Orientierung an der DIN 33430 werden erste Vorschläge unterbreitet, die zur Professionalisierung des Coachings im Unternehmenskontext beitragen.

Schlüsselwörter Coach-Auswahl · Coach-Assessment · Eignungsdiagnostik · Auswahlverfahren · closed shop · Anforderungen · DIN 33430

\section{Coach Selection by Companies-Ritualized Self-Assurance in the Closed Shop?}

\begin{abstract}
The increased demand for coaching as a personnel development measure in recent years results in a higher need for capable coaches, who have to be selected by the companies in advance. So far, little research has been done on how companies choose the right coaches and which requirement criteria is used as a basis. Based on initial findings, only quality surrogates are checked due to the lack of reliable requirement criteria. The lack of transparency in the market and the lack of verifiability of certificates and professional programs are two possible causes.
\end{abstract}

Louisa Schilling

Louisaschilling.hmkw@gmail.com
1 Hochschule Fresenius, Köln, Deutschland 
The present article addresses this question and illustrates the strategic background, motives and procedures of all stakeholders involved in the selection of coaches using an empirical qualitative interview study. Coaches, company representatives and service providers, who undertake the coach selection instead, are interviewed and their statements are compared for a global view on the market. Looking at the results it becomes apparent that the selection of coaches is still a business of relation and recommendation and therefore a closed shop. The poorly standardized selection interviews and the predominance of competence assumption and gut feeling as selection criteria suggest that the requirements of aptitude diagnostics are not reached yet. For the improvement of the selection processes and a stronger orientation towards the DIN 33430 primary propositions are given which contribute to the professionalization of coaching in the context of companies.

Keywords coach selection $\cdot$ coach-assessment $\cdot$ aptitude diagnostics $\cdot$ selection process $\cdot$ closed shop $\cdot$ requirement criteria

\section{Einleitung}

Coaching hat sich in den letzten Jahren als Personalentwicklungsmaßnahme zunehmend etabliert (Gross und Stephan 2015). Dies lässt auf einen zunehmenden Bedarf der Unternehmen an kompetenten Business-Coaches schließen, welcher den Einsatz von elaborierten Auswahlroutinen fordert, damit sinnvolle Matchingprozesse stattfinden und gescheiterte Prozesse vermieden werden. Im Unterschied $\mathrm{zu}$ den meisten Personalauswahlentscheidungen ist die Entscheidung für einen Coach schwieriger und unsicherer. Denn die Coaching-Beziehung ist vertraulich, temporär und hochgradig individuell. Der Erfolg hängt vom Zusammenwirken mehrerer Parteien - Klient, Coach und Organisation - ab (Koproduktion) und ist als immaterielle Dienstleistung schwierig zu bewerten (Greif 2015). Da der Coach als in der Regel externer Dienstleister nicht in die reguläre Arbeitsorganisation eingebunden ist, kann er lediglich punktuell und indirekt durch seinen Einfluss auf den Klienten wirksam werden (Stephan und Gross 2011; Greif 2015). Neben den von Klienten und/oder Unternehmen intendierten positiven Wirkungen sind allerdings auch unbeabsichtigte (positive wie negative) Wirkungen von Coaching denkbar und inzwischen empirisch belegt (Schermuly 2018; Schuler 2019). Eine schlechte oder unsachgemäße Personalauswahl in der Coach-Rekrutierung fehlt allerdings in der Aufstellung. Es muss von einer Intransparenz und Unklarheit über die Ausbildung und Kompetenz von Coaches ausgegangen werden (Kaul und Webers 2009). Aufgrund dieser Merkmale und Umstände, und weil Coaching eine teure Personalentwicklungsmaßnahme ist, erweist sich das Thema CoachAuswahl als höchst sensibles Thema für Unternehmen.

Wie gestaltet sich also bislang die Coach-Auswahl in Unternehmen? Welche Verfahren kommen hierbei zum Einsatz und inwiefern könnte diese ggf. verbessert werden? Der Beantwortung dieser Fragen widmet sich die vorliegende Studie.

\section{Coach-Auswahl als eignungsdiagnostisches Problem}

Schon vor geraumer Zeit hat Kühl das Thema Coach-Auswahl auf der Basis von mehr als 20 Experteninterviews dargestellt und kritisiert. Seine These lautet: „Weil der Coaching-Markt durch die Anbieter sehr wenig strukturiert wird und von Anbieter-Seite kaum Qualitätskontrollen durchgesetzt werden, findet die Kanalisierung über die Personalentwickler der Organisationen statt“ (Kühl 2006; S. 90). Diese regulieren die Anbieterseite durch die Einrichtung von Coach-Pools. Zugleich positionieren sie sich auf diese Weise anspruchsvoll und mächtig gegenüber den eigenen Führungskräften (Kühl 2008a). Die neue Macht der Personalentwickler als „Gatekeeper“ sei allerdings relativ: „Das Dilemma der Personalentwicklung ist, dass sie, wie auch viele andere Tätigkeiten in Organisationen - siehe nur Management -, selbst nur sehr begrenzt professionalisiert ist. Sie gewinnt an Einfluss, es ist aber unklar, ob sie ihr Leistungsversprechen einlösen kann“ (Kühl 2006; S. 91). Das Thema Evaluation werfe sie in ein kaum lösbares Dilemma: Entweder verweigerten sie sich der Evaluationsfrage (Vertraut uns, wir sind die Experten!). Oder sie verfielen in eine organisationale Heuchelei, behaupteten voreilig Effekte, die sich bei näherer Betrachtung als fragwürdig erwiesen. In beiden Fällen würden sie ihren professionellen Status selbst wieder untergraben, so Kühl (2008a).

Im Zuge der Untersuchung der Auswahlprozesse von Coaches lässt sich auch die Verhandlungssituation zwischen dem Anbieter (Coach) und dem Nachfrager (Personalentwicklung) beschreiben. Stephan und Gross (2011) beschreiben diese mittels der Agency-Theorie aus der Neuen Institutionenökonomik. Diese Theorie fokussiert insbesondere Informationsasymmetrien zwischen Nachfragern (Principal) und Anbietern (Agents). Diese Informationsasymmetrien entstehen im Coaching-Kontext durch die bereits zum Teil beschriebenen Eigenschaften des Coachings als wissensintensive Dienstleistung, z. B. durch die Immaterialität und Simultanität, die Integration eines externen Faktors (dem Klienten selbst) und die Heterogenität der Dienstleis- 
tung, wobei prinzipiell ein Informationsvorsprung der Coaches (Agents) anzunehmen ist (Gross und Stephan 2011). Die Autoren beschreiben diesen Informationsvorsprung als „Gefahr für die Nachfrageseite, einen Coaching-Anbieter mit mangelnden Qualifikationen (,Hidden Characteristics“), falschen Absichten (,Hidden Intentions') oder verdeckten Motivationsdefiziten zu akquirieren“ (Stephan und Gross 2011, S. 14). Doch auch Anbieter können falsche Erwartungen über die Absichten des Unternehmens, die zu leistende Tätigkeit und die damit verbundenen Anforderungen entwickeln. „Die für die Auswahl ausschlaggebenden Kriterien sind für den Coach meist nicht transparent. Er oder sie hat häufig keine Informationen über die hidden agen$d a$ [Hervorhebung i. O.] des Unternehmens." (Nyuli 2016, S. 417). Es können im konkreten Fall diverse Aspekte den Ausschlag für einen Einsatz gegen, bspw. äußerliche Merkmale, Kompetenzvermutungen, Branchenkenntnis oder eine Empfehlung. Um diese Informationsasymmetrien zu überbrücken, empfiehlt die Agency-Theorie Screening-Maßnahmen (Auswahlverfahren) für die Nachfrage-Seite und Signalling-Maßnahmen für den Anbieter, das bedeutet freiwillig Informationen zu kommunizieren, also Eigenschaften zu signalisieren.

Prinzipiell ist die Agency-Theorie durch Misstrauen auf beiden Seiten gekennzeichnet. Zumindest verlangt sie erhöhte Aufmerksamkeit der Verhandlungspartner, damit die Wahrscheinlichkeit von Missverständnissen oder Irrtümern gering bleibt. Coaching ist allerdings kein einfach zu spezifizierendes Produkt, sondern eine Dienstleistung. Es handelt sich um ein Erfahrungs- und Vertrauensgut (Stephan und Gross 2011; Greif 2015). Beide Verhandlungspartner benötigen ein reflektiertes Wissen über den Auswahlprozess und die erfolgskritischen Kriterien. Kühl (2008c) bezweifelt diese Kompetenz insbesondere auf Unternehmensseite. In Ermangelung belastbarer Auswahlkriterien bediene man sich sog. Qualitätssurrogate, also Ersatzkriterien, welche die Qualität wiederspiegeln sollen, zum Beispiel das Alter des Coaches (Seniorität) oder seine Führungserfahrung, wie auch Webers (2013) mutmaßt. Der Auswahlprozess von Coaches für Unternehmen vollziehe sich demnach oft als ritualisiertes Geschehen - frei nach dem Märchenmotto „Des Kaisers neue Kleider“ (Kühl 2008b). Man verlange zwar Kompetenzausweise, prüfe diese allerdings nicht (ausreichend): Coach-Auswahl als Farce.

\subsection{Forschungsstand zur Auswahl von Coaches im Unternehmenskontext}

Insgesamt ist die Auswahl von Coaches noch kein intensiv beforschtes Feld, so findet man bisher nur vereinzelt empirische Studien, unter denen die im Panel-Design durchgeführten sog. Marburger Coaching-Markt-Studien der Jahre 2009, 2011 und 2013 (DBVC 2013) hervorstechen. Dem- nach verläuft die Anbahnung der Zusammenarbeit von Coaches und Unternehmen in erster Linie als Empfehlungs- und Beziehungsgeschäft. Knapp $75 \%$ der im Rahmen der sog. Marburger Coaching-Markt-Studien Befragten (Gross und Stephan 2015) geben an, dass Empfehlungen sehr wichtig sind. Besonderes Gewicht hierbei haben offenbar die Referenzen von Coaches aus dem unternehmenseigenen CoachPool (sehr wichtig: 38\%). Einen Coach-Pool zu unterhalten berichten $53 \%$ der befragten Unternehmen.

Als Auswahlkriterien nennen die Unternehmen vor allem Referenzen und positive Empfehlungen von Dritten mit dem Coach (sehr wichtig: 62,7\%), Coaching-Erfahrung in Jahren (sehr wichtig: 55,2\%), Schwerpunktkompetenzen (sehr wichtig: 50,7\%), Ausbildung als Coach (sehr wichtig: 50,0\%), ethisches Selbstverständnis (sehr wichtig: 49,3\%). Eine Neuauflage der sog. Marburger Coaching-Markt-Studie (Stephan und Rötz 2018) erreicht zwar insgesamt eine geringere Stichprobengröße, bezüglich der Auswahlkriterien für die Aufnahme in einen Coach-Pool sind die Stichproben aber vergleichbar. Tab. 1 zeigt die Ergebnisse im Vergleich der Jahre 2009 und 2018.

Obwohl Empfehlungen und Referenzen bei der Anbahnung des Kontakts als sehr wichtig bezeichnet werden, im Jahr 2018 sogar von 80,3\% der befragten Unternehmen, findet sich dieses Kriterium im Hinblick auf die Aufnahme in einen Coach-Pool lediglich auf Rangplatz 4. Auffällig ist, ohne nun die Daten im Einzelnen interpretieren zu wollen, wie wenig wichtig den Unternehmen eine Verbandszertifizierung ist: Lediglich ungefähr die Hälfte der Befragten äußert sich entsprechend.

Der Auswahlprozess gestaltet sich meist mithilfe von zwei- bis dreistündigen Vorstellungsgesprächen bzw. einem persönlichen Kennenlernen (Stephan und Gross 2011). Strukturierte Auswahlverfahren mit mehreren Bewerbern, die Prüfung von schriftlichen Unterlagen, Assessment-Center und Arbeitsproben sind weniger verbreitete Vorgehensweisen. Wichtig in den Auswahlverfahren ist vor allem das Vertrauen, welches sich zwischen Coach und Unternehmensvertretern als „Bauchgefühl“ entwickelt (Nyuli 2016). Durch die Empfehlungen von Dritten, denen die Unternehmensvertreter vertrauen, erhalten die Coaches einen Vertrauensvorschuss, der durch die Wahrnehmung der vertrauensbildenden Aspekte im Gespräch geprüft wird (Oellerich et al. 2013).

Tonhäuser (2012) befragte Personalentwicklungsverantwortliche deutscher Großunternehmen $(N=104)$. Demnach hat die Mehrheit der Unternehmen einen Coach-Pool gebildet $(86,8 \%)$, Qualifikationsstandards für Coaches definiert $(76,8 \%)$ sowie Ablaufstandards für Coaching-Prozesse festgelegt $(69,7 \%)$. Als Kriterien für die Coach-Auswahl berichtet sie: Berufserfahrung, Vertrauenswürdigkeit und Integrität, Persönlichkeit und soziale Kompetenzen (jew.: $100 \%)$, Coaching-Ausbildung (97\%), breites und trans- 
Tab. 1 Ergebnisse zu den Auswahlkriterien für die Aufnahme eines Coachs in einen Coach-Pool im Vergleich; Auszüge (Gross und Stephan 2015, S. 9; Stephan und Rötz 2018, S. 24)

\begin{tabular}{lllll}
\hline & \multicolumn{3}{l}{ Gross und Stephan $(2015)(N=67)$} & \multicolumn{2}{l}{ Stephan und Rötz $(2018)(N=61)$} \\
\cline { 2 - 5 } & Sehr wichtig $(\%)$ & Wichtig $(\%)$ & Sehr wichtig $(\%)$ & Wichtig $(\%)$ \\
\hline Ausbildung & 50,0 & 44,1 & 59,0 & 39,3 \\
Coach-Berufserfahrung & 55,2 & 41,8 & 57,4 & 36,1 \\
Schwerpunktkompetenzen & 50,7 & 43,3 & 57,4 & 34,4 \\
Referenzen & 62,7 & 31,3 & 59,0 & 29,5 \\
Ethisches Selbstverständnis & 49,3 & 44,8 & 50,8 & 37,7 \\
Kosten/Preis & 13,6 & 63,6 & 19,7 & 54,1 \\
Führungserfahrung & 29,9 & 47,8 & 31,1 & 34,4 \\
Branchenerfahrung & 20,9 & 53,7 & 24,6 & 45,9 \\
Regionale Nähe & 16,7 & 45,5 & 21,3 & 37,7 \\
Verbandszertifizierung & 14,1 & 35,9 & 18,0 & 34,4 \\
\hline
\end{tabular}

parentes Methodenspektrum, transparentes Coaching-Konzept (jew.: 95\%), psychologische Kenntnisse (94\%), Referenzen und Reputation (91\%), Management- und Führungserfahrung (86\%) sowie weitere Aspekte.

Finke und Kanning (2019) befragten jüngst 257 Unternehmensvertreter zum Thema Coach-Auswahl. Die Reputation des Coachs und die Empfehlungen durch Dritte stehen hier wieder an der Spitze als wichtige Anforderungen an Coaches. Allerdings werden Berufs-, Lebens- und Branchenerfahrungen ebenfalls sehr geschätzt. Des Weiteren werden verstärkt konkrete Kompetenzen (Beratung, Methoden, Coaching, Psychologie) erwartet. Die Unternehmen fordern in hohem Maße Referenzen $(77,4 \%)$ und Arbeitsbeispiele $(72,4 \%)$ ein und erwarten die Vorlage eines Coaching-Ausbildungszertifikats $(66,5 \%)$.

Bezüglich der diagnostischen Methoden, mit denen die Unternehmen die erwünschten Kriterien abprüfen wollen, dominiert die Selbstpräsentation des Coachs $(60,7 \%)$. Während größere Unternehmen ( $>500$ Mitarbeiter) zudem stärker auf teilstrukturierte Interviews setzen $(61,9 \%)$, herrscht in den kleineren Unternehmen eher das unstrukturierte Interview vor $(55,3 \%)$. Arbeitsproben $(21,4 \%)$, Fallstudien/ Rollenspiele (16\%) oder Assessment-Center (7\%) werden bislang eher selten eingesetzt. Die Autoren resümieren ihre Befunde skeptisch: ,Vor die Wahl gestellt, einen Coach einzustellen, der eine eindrucksvolle Kundenliste aufweist und persönlich empfohlen wird, obwohl er über keine qualifizierende Ausbildung verfügt, oder einen ausgebildeten Coach mit weniger Erfahrung einzukaufen, würden sich viele Unternehmen wohl für den ersten Kandidaten entscheiden“ (Finke und Kanning 2019; S. 53).

Anderweitige Untersuchungen sind kaum repräsentativ und methodisch fragwürdig (Müller 2012; Müller et al. 2015; Baumann 2016). Schließlich wird auch vereinzelt von Bemühungen des Outsourcings der Coach-Auswahl an externe Dienstleistungsunternehmen berichtet (Reimer 2010; Reimer und Fengel 2010). Neuerdings treten auf dem Markt zudem Plattformunternehmen auf, die sich zwischen Coach und Unternehmen schalten (Bachmann und Fietze 2018). Sie begründen das Matching von Coach und Klient mittels eines Algorithmus, der allerdings nicht offengelegt wird.

Der schon von Kühl (2008a) geäußerte Mauscheleiund Closed-Shop-Verdacht steht damit weiterhin im Raum. Auch wenn es erste Ansätze für die Verbesserung der Auswahl von Coaches gibt (Kaul 2005, 2008; Henle 2009; Steinle et al. 2009; Scholer und Lendner 2012; Stiftung Warentest 2014), offenbart sich im Vergleich zum professionellen Vorgehen (DIN 33430; DIN 2014) deutliches Potenzial, wie schon Webers (2015) feststellte.

\subsection{Eignungsdiagnostik - die DIN 33430}

Die erstmals im Jahr 2002 veröffentlichte Norm DIN 33430 (DIN 2014) stellt die Anforderungsanalyse an den Beginn jeder Personalauswahlentscheidung. Auf deren Basis ist ein Auswahlprozess zu entwickeln, in dem geeignete Instrumente, nämlich solche, welche angemessene Aussagen zu den geforderten Merkmalen treffen können, von geeigneten Personen einzusetzen sind. Eine Evaluation der Auswahlentscheidung schließt diesen Prozess ab.

- Anforderungsanalyse: Sie umfasst die Ermittlung berufsrelevanter Voraussetzungen mit dem Ziel, relevante Informationen (Kriterien) bereitzustellen. Die Perspektive der Beschreibung von Situationen und Tätigkeiten (Arbeitsanalyse) und von Personenmerkmalen (Anforderungsanalyse) beziehen sich dabei aufeinander. Methodisch lässt sich zwischen verschiedenen, eher analytischen oder empirischen (Experten- vs. Mitarbeiterurteil), Varianten unterscheiden.

- Auswahl: Die Identifizierung von Kandidaten, die die Kriterien erfüllen, geschieht über den Einsatz von Verfahren. Hier sind nach Schuler (2014) drei Wege (trimodaler Ansatz) zu unterscheiden: 1. Die Betrachtung über- 
dauernder Eigenschaften, die mittels Testverfahren (z. B. Intelligenz) erhoben werden. 2. Die Betrachtung von bewältigten Arbeitsaufgaben, welche sich über die Analyse der Biografie zeigen. 3. Die Betrachtung von konkretem Verhalten, das sich über Simulationen oder Arbeitsproben erschließt. Nur eine Verknüpfung aller drei Methoden liefert ein ganzheitliches Bild, denn überdauernde Eigenschaften müssen sich nicht in konkreten Situationen zeigen und vergangene Arbeitserfahrungen müssen nicht zu neuen Verhaltensanforderungen passen.

- Entscheidung und Evaluation: Da Entscheidungen immer fehlerbehaftet sind, sollen sie im Weiteren evaluiert werden, um die Entscheidung zu bestätigen oder zu revidieren sowie das Entscheidungsverfahren selbst zu verbessern.

Im Prozess der Beurteilung sind zudem drei aufeinander bezogene Qualitätsdomänen zu unterscheiden:

- Organisationsqualität (Kontext): Ausgangsbasis einer berufsbezogenen Eignungsbeurteilung muss ein klares Anforderungsprofil sein. Kompetente Anwender sollen auf dieser Basis mit geeigneten Produkten nachvollziehbare (evaluierbare) Entscheidungen treffen. Die Objektivität der Durchführung der Verfahren ist zu gewährleisten. Es sind daher geeignete (Standard-)Prozesse zu definieren. Die Dokumentation der Instruktionen, Verfahrenselemente sowie der Einstufungshilfen und Regeln für die Ableitung der Eignungsbeurteilung sind unerlässlich.

- Produktqualität (Instrumente): Für die berufsbezogene Eignungsbeurteilung dürfen nur solche Verfahren (Produkte/Instrumente) verwendet werden, die nachweislich einen Bezug zu den Anforderungen haben. $\mathrm{Zu}$ jedem Verfahren der Eignungsbeurteilung ist darzulegen, wie es $\mathrm{zu}$ handhaben ist (Handbuch). Der Anwender muss in die Lage versetzt werden, das Verfahren kritisch zu bewerten und ordnungsgemäß anzuwenden.

- Personenqualität (Anwender): Diese müssen über fundierte Kenntnisse von Eignungsbeurteilungen und möglichst angeleitete Praxiserfahrungen in Entwicklung, Planung, Gestaltung und kontrollierter Durchführung von Verfahren zur Eignungsbeurteilung sowie deren Evaluation verfügen. Sie übernehmen die Fachaufsicht und müssen sicherstellen, dass alle von ihnen eingesetzten Mitwirkenden aufgabenspezifisch geschult und eingewiesen werden.

Der Erfolg einer Eignungsbeurteilung hängt von allen Domänen gleichermaßen ab. Ein erfahrener Anwender ohne Anforderungsprofil weiß nicht, wen er suchen soll. Nutzt er die passenden Auswahlverfahren nicht, fehlen belastbare Daten. Die DIN 33430 hat sich etabliert, konstatiert Kersting (2009). Jüngst legte das Diagnostik- und Testkurato- rium, ein vom Berufsverband Deutscher Psychologinnen und Psychologen e. V. und von der Deutschen Gesellschaft für Psychologie e.V. getragenes Gremium und zuständig für alle Aspekte der Qualitätssicherung und Qualitätsoptimierung, ein Grundlagenwerk zur Eignungsdiagnostik nach DIN 33430 vor (2018). Der Blick auf die bislang vorliegenden Studien zur Coach-Auswahl, bspw. Stephan und Gross (2011), weckt jedoch den Verdacht, dass die Anforderungen an eine professionelle Eignungsbeurteilung in der Praxis der Coach-Auswahl bislang wenig erfüllt werden.

\section{Ziel der Untersuchung}

Wie bereits dargelegt, sind die Erkenntnisse über die CoachAuswahl in deutschen Unternehmen noch lückenhaft. Insbesondere fehlt es an Einsichten in strategische Hintergründe, das heißt Motive, Absichten und Denkweisen der beteiligten Akteure. Im Rahmen dieser Studie wird daher folgenden Forschungsfragen nachgegangen:

1. Wie gestaltet sich die Auswahl von externen Coaches in deutschen Unternehmen aus den unterschiedlichen Perspektiven der Beteiligten und inwiefern erfolgt diese nach eignungsdiagnostischen Standards?

2. Welche Verbesserungsbedarfe werden sichtbar und wird der Bedarf nach standardisierten Verfahren geäußert?

3. Welche Anforderungen werden an Coaches gestellt?

4. Wie verbreitet sind externe Dienstleistungen um die Coach-Auswahl (Makler) und wie werden sie bewertet?

In dieser Forschungsperspektive wird ein Alleinstellungsmerkmal der vorliegenden Studie deutlich. Sie soll erste Einblicke darin gewähren, wie die Dienstleistungen von allen beteiligten Marktteilnehmern - externen Coaches, betrieblichen Einkäufern und externen Maklern - gesehen und bewertet wird.

\section{Methode}

Das Forschungsfeld wird bislang überwiegend durch quantitative Daten und kursorische Beschreibungen erhellt, die die Interessenslage der Akteure, ihre Strategien und Taktiken wenig beleuchten. Aus diesem Grund wurde ein qualitatives Studiendesign gewählt, welches in einer MultiStakeholder-Perspektive detaillierte und anschauliche Einblicke ermöglichen soll.

\subsection{Vorgehen}

Es wurde eine qualitative, halbstrukturierte Interviewstudie durchgeführt. Die Rekrutierung der Interviewpartner erfolgte als anfallende Stichprobe über persönliche und berufli- 
che Kontakte des Netzwerks der Autoren. Die Interviewpartner setzten sich aus drei Unterstichproben zusammen: Unternehmensvertreter, Coaches und externe Coach-Makler. Die insgesamt 23 leitfadengestützten Interviews hatten eine durchschnittliche Dauer von 47 min und wurden entweder face-to-face oder telefonisch durchgeführt sowie via Audioaufnahme dokumentiert. Die Datenerhebung fand im Zeitraum von Oktober bis Mitte November 2018 statt. Die gewonnenen verbalen Daten wurden anschließend vollständig transkribiert und mithilfe der qualitativen Inhaltsanalyse (Mayring 2015) ausgewertet.

\subsection{Instrumente}

Zur Interviewführung wurde ein Interviewleitfaden entwickelt, der für das semi-strukturierte Interview Leitfragen enthält und mit Ad-hoc-Fragen im Verlauf der Gespräche ergänzt wurde. Die einzelnen Leitfragen sind jeweils auf die Unterstichprobe adaptiert worden, sind inhaltlich aber identisch. Die Beispielfragen entstammen dem Interviewleitfaden für Unternehmensvertreter.

Der Einstieg in das Interview umfasst Fragen zur Person (z.B. „Welchen Bezug haben Sie zum Thema Coaching?") und zur Organisation (z.B. ,Welche Verbreitung hat Coaching als Entwicklungsmaßnahme in Ihrem Unternehmen?“), fokussiert dann auf die Implementierung von Coaching in Organisationen (z.B. ,Wer ist für das Thema Coaching intern zuständig? Und welche Qualifikation besitzen diese Personen?") und schließlich auf die Auswahl von Coaches (z. B. ,Welche Methoden oder Instrumente setzen Sie ein, um zu überprüfen, ob der Coach zu Ihnen bzw. der Organisation passt?" ).

Neben der Abfrage des Status quo der Auswahl wird in einem weiteren Schritt erfragt, wie die Auswahl optimiert werden kann (z. B. ,Was fehlt Ihnen für die valide Auswahl von Coachs? Was bräuchten Sie dazu?“) oder zukünftig aussehen sollte.

Fragen zu den Anforderungen an Coaches (z. B. „Welche Anforderungen, Kenntnisse, Qualifikationen und Fähigkeiten bringt der Coach mit, der bei Ihnen erfolgreich ist?") sowie zur Gesamteinschätzung des Marktes (z. B. ,Wie bewerten Sie als interner Experte den Coaching-Markt in Deutschland und wie finden Sie sich als Kunde in diesem Markt zurecht?") und bestimmter spezifischer Dienstleistungen um die Coach-Auswahl (z. B. ,,Was ist Ihre Meinung als potenzieller Kunde zu den Drittanbietern, z. B. Verbände, Netzwerkorganisationen, Dienstleister, die Leistungen um die Suche und Vermittlung von Coachs anbieten?“) runden den Fragenkatalog ab.

\subsection{Stichprobe}

Die Stichprobe besteht aus insgesamt 23 Interviewpartnern. Neun Interviewpartner sind der Unterstichprobe der Coaches zuzuordnen (5 Männer und 4 Frauen), 13 Interviewpartner (6 Männer und 7 Frauen) ergeben die Unterstichprobe der Unternehmensvertreter. Lediglich eine weibliche Interviewpartnerin ist der Unterstichprobe der Coach-Makler zuzuordnen. Im Laufe der Stichprobenakquisition erweist sich dieses Feld als schwer zugänglich; die Kontaktanfragen werden teils abgelehnt oder unbeantwortet gelassen. Die Gründe für die schwierige Zugänglichkeit und die mögliche Deutung und Auswirkung dieser Tatsache werden im Zuge der kritischen Würdigung näher erläutert.

Die Unterstichprobe der Coaches ist in ihren Merkmalsausprägungen relativ homogen. Alle befragten Coaches verfügen über eine oder mehrere Coaching-Ausbildungen mit systemischem Hintergrund und mehrjährige Erfahrung als Coach (acht von neun Coaches mit fünf oder mehr Jahren Erfahrung). Zielgruppe der angebotenen Leistungen sind bei allen Coaches Führungskräfte. Sieben der Coaches verfügen über einen Erfahrungshintergrund im Wirtschaftskontext und sind selbst in verschiedenen Funktionen, oft auch in Führungsrollen in Unternehmen aus unterschiedlichen Branchen, meist Konzernen, tätig (gewesen). Die Qualifikation der Coaches variiert: Vier der Coaches sind DiplomPsychologen, ein Coach ist zudem als psychologischer Psychotherapeut approbiert. Der Vertreter der Unterstichprobe Coach-Makler ist ebenfalls selbst seit mehr als 20 Jahren Coach und neben der Beratung von Unternehmen zudem Autor.

Die befragten Vertreter der Unternehmen sind seit mindestens zwei Jahren in ihrer aktuellen Funktion im Unternehmen tätig. Lediglich ein Interviewpartner ist erst seit wenigen Monaten in seiner aktuellen Position. Acht der 13 Unternehmensvertreter haben selbst eine Coaching-Ausbildung absolviert bzw. befinden sich zurzeit noch in Ausbildung. Der Tätigkeitsbereich aller Unternehmensvertreter ist dem Personalwesen zugeordnet. Die Größe der Unternehmen, in denen die Interviewten tätig sind, variiert stark. Alle Organisationen sind als Großunternehmen bzw. Konzerne zu bezeichnen, die Mitarbeiterzahlen liegen zwischen knapp 2000 und 200.000 Mitarbeitern; der Median liegt bei circa 18.000 Mitarbeitern. Auch im Hinblick auf Branchen gestaltet sich die Stichprobe als vielfältige Unternehmenslandschaft. Zwei Unternehmen sind dem Handel zuzuordnen, zwei weitere sind im Bereich der Forschung und Entwicklung tätig. Darüber hinaus sind zwei Industrie-Unternehmen in der Stichprobe vertreten. Die Finanzbranche, Logistik, Technologie, Energie und Beratung sind ebenfalls durch jeweils ein Unternehmen in der Stichprobe repräsentiert. Bei einem Unternehmen handelt es sich um einen Wohlfahrtsverband. Lediglich ein Unternehmen (For- 
schung und Entwicklung) hat die Rechtsform einer Körperschaft des öffentlichen Rechts. Die übrigen Unternehmen entstammen der Privatwirtschaft.

\section{Ergebnisse}

Im Folgenden werden die Ergebnisse der qualitativen Interviewstudie anhand der einzelnen Forschungsfragen dargestellt.

\subsection{Auswahl von Coaches in deutschen Unternehmen}

Coaching ist ein Beziehungs- und Empfehlungsgeschäft. Dies geht sowohl aus den Aussagen von Coaches, als auch aus den Berichten der Unternehmensvertreter hervor. Dabei wird diese Tatsache häufig wenig kritisch beurteilt bzw. auf Gefahren oder Risiken geprüft. Vereinzelt wird festgestellt, dass es zu einem Selektionseffekt, also der Bevorzugung Einzelner sowie der Nicht-Beachtung von neuen oder unbekannten Coaches kommt. Die Empfehlung durch Andere stellt das benötigte Eingangstor für den Coach dar, welches aus der Natur des Marktes heraus nicht gegeben ist. Ein weiterer, in den befragten Unternehmen und auch bei den befragten Coaches populärer Weg der Anbahnung ist die bereits bestehende Zusammenarbeit zwischen beiden Parteien. Das Risiko, über Empfehlungen eine Negativ-Auslese zu treffen, wird von den Interviewten als relativ gering eingeschätzt, es herrscht ein hohes Vertrauen in die Qualität der empfohlenen Coaches.

Der eigentliche Auswahlprozess wird von den Interviewpartnern relativ unterschiedlich beschrieben. Ein dreischrittiges Vorgehen mit Sichtung der Unterlagen und Profile, ein telefonisches Vorgespräch und ein persönliches Kennenlernen stellt eine verbreitete Herangehensweise von Unternehmen dar. Das persönliche Gespräch mit dem potenziellen Coach suchen zwölf der 13 Unternehmen. Die befragten Coaches hingegen berichten vereinzelt von ausführlicheren Verfahren: Drei der Coaches haben bereits in der Vergangenheit an einem Assessment-Center teilgenommen. Coach-Assessments sind aber nach Auskunft der Coaches eine eher seltene, wenig verbreitete Form der Auswahl. Der Vertreter der Coach-Makler vertritt schließlich die vollständige Outsourcing-Position und ist der Meinung, dass die Implementierung von Coaching in der Organisation durch Dienstleistungsunternehmen begleitet werden sollte, weil die Verantwortlichen in den Unternehmen aufgrund ihrer Position in der Hierarchie nicht in der Lage seien, Coaching-Konzepte passend zu den Bedarfen im Unternehmen zu entwickeln und umzusetzen (Interview 4, Schilling 2019; S. 46). Die Suche und Auswahl ist aus der Sicht des Dienstleistungsvertreters aufgrund der Marktbedingungen ein schwieriges Unterfangen für die Coaching-Verantwortlichen: „Das ist aussichtlos [...]. Also wie soll das gehen?! Alle lügen, manipulieren, dominieren im Internet. Wie soll das gehen?! Auf jeden Fall an jemanden wenden, der was davon versteht." (Interview 4, Schilling 2019; S. 52).

Persönliche Auswahlgespräche sind das Instrument der Wahl. Die Gespräche haben eine Dauer von einer halben Stunde bis zwei Stunden und werden durch den oder die zuständigen HR-Verantwortlichen durchgeführt. Der Strukturierungsgrad der Auswahlgespräche differenziert wie die Inhalte ebenfalls, grundsätzlich geben aber fast alle interviewten Unternehmensvertreter an, dass die Standardisierung der eigenen aktuellen Vorgehensweise relativ gering ist. Das Interesse und Ziel der Unternehmensvertreter für diese Auswahlgespräche liegen darin, den Coach als Person kennenzulernen, relevante Anforderungen und Erfahrungen des Coachs zu untersuchen und die Passung zu prüfen. In den Aussagen der Coaches lassen sich darüber hinaus noch der Aufbau von Vertrauen und Atmosphäre als Zielsetzung des Gesprächs auffinden. Dies drückt das Beziehungsinteresse der Coaches aus, die in solchen Situationen eine Beziehung zum Ansprechpartner im Unternehmen herstellen wollen. Versuche, den Beziehungsaspekt des Gesprächs durch strikt standardisierte Fragen auszuklammern, wird von einem Coach sogar explizit kritisiert: „Das so komplett auszublenden finde ich schwierig, weil darauf kommt es ja an [...] Und das kann ich ja auch nicht mit [...] objektiv messbaren diagnostischen Mitteln sagen [...] versuchen numerisch irgendwie darzustellen." (Interview 20, Schilling 2019; S. 252).

Der Coach-Makler sowie zwei der befragten Unternehmen führen außerdem Simulationen durch, welche als besonders erhellend und hilfreich von diesen wahrgenommen werden. In den Unternehmen, in welchen keine Simulationen durchgeführt werden, werden diese abgelehnt. Auch unter den befragten Coaches teilen sich die Meinungen zum Einsatz von Simulationen. Bei professioneller Gestaltung lehnt ein Coach die Durchführung nicht ab, andere äußern sich aber kritisch über die Künstlichkeit und die Abwesenheit von tatsächlich Betroffenen sowie den Test-Charakter der Situation, welche mit Druck verbunden sei und verhindere, den Coach wirklich kennenzulernen.

Insgesamt zeigen sich die interviewten Coaches Auswahlverfahren gegenüber offen. Es wird aber vereinzelt betont, dass bei einer langjährigen Tätigkeit als Coach ein solch strukturiertes Verfahren nicht notwendig sei und durch ein professionell geführtes Interview ersetzt werden könne: „Und ob ich dann jetzt noch so ein AC brauche, (...) da würde ich sagen, wenn jemand seit 15 Jahren nachweislich tätig ist und sonst sowas nachweisen kann, dann kann ich mir das ersparen." (Interview 3, Schilling 2019; S. 34). Auch an anderen Stellen wird deutlich, dass standardisierte und mit (zeitlichem) Aufwand verbundene Verfahren bei 
Coaches nicht unbedingt auf große Akzeptanz stoßen bzw. Hürden darstellen.

Einige Interviewpartner, sowohl Coaches als auch Unternehmensvertreter, stellen die Bewertbarkeit des Coachs und damit die allgemeine Sinnhaftigkeit von Auswahlverfahren infrage. Die Informationsfunktion sei in den Auswahlverfahren zwar erfüllt, am Ende komme es aber auf die individuelle Passung zum Klienten und die Beziehung an, die sich mithilfe diagnostischer Mittel und einem numerischen oder anders gearteten Bewertungssystem nicht erfassen und darstellen lasse: „Aber ich würde nicht mir anmaßen wollen, dass man das komplexeste System der Welt, den Menschen, innerhalb einer Stunde erfassen könnte" (Interview 16, Schilling 2019; S. 199). Passend zur schwierigen, fast unmöglichen Beurteilung von Beziehung und Passung zum Unternehmen und zum potenziellen Klienten sowie der Zielsetzung, ein Gefühl für den Coach zu gewinnen, wird in einigen Gesprächen auch das Bauchgefühl, der gesunde Menschenverstand oder das Vertrauen als Indikator für die Auswahlentscheidung erwähnt: „Es ist eben (...) einfach ein Stückweit Bauch, was eben bei so einer Auswahl eben dann auch eine Rolle mitspielt" (Interview 17, Schilling 2019; S. 214).

Schließlich zeigen sich unterschiedliche Ausprägungsformen der Coach-Pools bei sieben Unternehmen. Die meisten Pools haben einen Sättigungsgrad erreicht, die Erweiterung des Pools geschieht daher eher zufällig und recht selten. Bei zwei Unternehmen ist es in der jüngsten Vergangenheit zu einer Neustrukturierung des Pools gekommen, bei der alle aktuellen Coaches auf den Prüfstand gestellt wurden. Ein größerer Durchmischungsgrad durch Neubesetzungen ist in diesem Zuge aber nicht realisiert worden.

\subsection{Verbesserungsbedarfe der Auswahlprozesse}

Zunächst lässt sich feststellen, dass die von den Interviewpartnern genannten Verbesserungsbedarfe tendenziell kleineren Umfangs sind und es ein eher geringes Interesse an Veränderung gibt. Sowohl die Coaches (4 von 9) als auch die Unternehmensvertreter (9 von 13) sehen kaum oder keinen Anlass für Veränderung und stellen in den vergangenen Jahren eine positive Entwicklung in Richtung Professionalisierung der Coach-Auswahl fest. Die fehlende Veränderungsnotwendigkeit bzw. -motivation wird häufig auch mit der geringen Priorität der Auswahlprozesse begründet. Konkrete Veränderungsaspekte sind nach Angaben der Interviewpartner aus den Unternehmen die verstärkte zeitliche Auseinandersetzung mit dem Thema Coach-Auswahl, die Ergänzung der Gespräche um situative Fragen und die Verwendung eines strukturierten Gesprächsleitfadens. Auf Seiten der Coaches werden ähnliche Aspekte genannt. Darüber hinaus teilen sich die Meinungen, ob eine stärkere
Systematik und Standardisierung verfolgt werden soll (Assessments) oder ob Unternehmen sich vermehrt auf ihre Intuition in der Auswahl verlassen sollen: „Ich (...) ganz persönlich glaube, es braucht kein strukturiertes Assessment.“ (Interview 8, Schilling 2019; S. 105); „Ich könnte mir tatsächlich vorstellen, dass hilfreich wäre, das Thema noch systematischer aufzustellen. Wenn ich jetzt so überlege, also es scheint tatsächlich noch nicht viele Assessment Center oder wirklich durchstrukturierte Interviews von Coaches zu geben." (Interview 1, Schilling 2019; S. 12) Alle weiteren genannten Veränderungsbedarfe beziehen sich bei beiden Unterstichproben nicht konkret auf die Auswahl, sondern auf die ganzheitliche Implementierung von Coaching in den Unternehmen, z. B. Verbesserung des Contractings und des Images sowie Steigerung der Akzeptanz. Coaches wünschen sich zudem die Ausweitung des Coaching-Angebots sowie eine aktivere Pflege des Coach-Pools, also u. a. eine aktivere Information über Unternehmensprozesse, Werte und Personalentwicklungsthemen.

Aufgrund der geringen Dichte an konkret genannten Optimierungspotenzialen für die Auswahlprozesse und einer allgemeinen Zufriedenheit mit den aktuellen Vorgehensweisen auf beiden Seiten entsteht der Eindruck, dass der Bedarf an Veränderung relativ gering ist. Insbesondere die kritischen Äußerungen der Coaches und Unternehmensvertreter im Zusammenhang mit standardisiertem Vorgehen weisen darauf hin, dass der Bedarf speziell für standardisierte Verfahren nicht allzu verbreitet ist. Die deutliche Präsenz von Verbesserungsvorschlägen im Hinblick auf die allgemeine Implementierung und Integration von Coaching in die Personalentwicklung der Unternehmen machen deutlich, dass in der Praxis allgemein noch andere, fundamentalere Entwicklungsfelder (z.B. Image und Akzeptanz, Zielsetzung und Zielgruppe usw.) bearbeitet werden müssen, bevor sich Gedanken um den Prozess der Auswahl der Coaches gemacht wird: „Wahrscheinlich müsste [...] ich mir die ganzen Prozesse ein bisschen genauer anschauen. Also wirklich von der Zielsetzung, [...] Bis hin zu welche [...] Kriterien stellen wir bei der Auswahl.“ (Interview 21, Schilling 2019; S. 273).

\subsection{Anforderungen an Coaches}

Insgesamt lässt sich bei der Betrachtung der Anforderungen an Coaches eine hohe Deckungsgleichheit feststellen. Die Wahrnehmung der Coaches, welche Kriterien für Unternehmen relevant sind oder sein könnten, ähnelt stark dem, was Unternehmen tatsächlich als Anforderung stellen. Die harten Anforderungen an Coaches, das heißt eindeutig messbare, objektive Kriterien, sind in erster Linie und nach Häufigkeit der Nennung die Erfahrung des Coaches im Wirtschafts- und Unternehmenskontext, die Ausbildung des Coachs, sowie die Führungserfahrung und die Erfah- 
rung in der Tätigkeit als Coach. Bei vergleichender Betrachtung fällt auf, dass Coaches die Kriterien Führungserfahrung und Branchen- bzw. Unternehmenserfahrung kritischer reflektieren als Unternehmensvertreter und darin auch Gefahren oder Risiken sehen. Trotzdem sind die Funktion und Sinnhaftigkeit dieser Kriterien für die Coaches einleuchtend und Zeichen für das Dilemma, in dem die Verantwortlichen stecken. Darin wird der Versuch gesehen, die Irrtumswahrscheinlichkeit in einem komplexen Markt gering zu halten: ,Das ist so ein Dilemma, was da auch Personaler haben. Die versuchen sich natürlich auch abzusichern. [...] Und dann versucht man sich dem anzunähern und versucht dann Kriterien zu finden, um eine gute Entscheidung zu treffen. Deshalb habe ich da vollstes Verständnis für" (Interview 20, Schilling 2019; S. 255). Ambivalent wird außerdem das Thema Zertifizierung und Verbandszugehörigkeit diskutiert. Relevanz hat für die meisten Unternehmensvertreter eher die Ausbildung selbst, nicht aber unbedingt ihre Zertifizierung über einen Verband, da über die Qualität der Zertifizierung keine Aussage getroffen werden kann. Auch Coaches sind sich hier uneinig. Einige begrüßen die Entwicklungen und werten diese als Professionalisierungsbemühung, anderen sind die Hürden zur Zertifizierung nicht anspruchsvoll genug und damit wenig aussagekräftig. Schließlich werden auch Referenzen als Anforderungskriterium genannt, die aus Sicht von Coaches und Unternehmen aber nicht oder nur in seltenen Fällen überprüft werden.

Darüber hinaus gibt es noch „weiche“ Anforderungen. Dies sind Merkmale, die sich nur subjektiv bewerten lassen, für die es naturgemäß zunächst keine Bewertungsskala gibt und die häufig kompetenz- und persönlichkeitsbezogen sind. An erster Stelle stehen hier die Anschlussfähigkeit des Coachs an das System seines Klienten und seine Seniorität. Letztere wird häufig am Alter gemessen, zum Teil werden an dieser Stelle auch Altersgrenzen kommuniziert. Für die befragten Unternehmensvertreter ist mit Seniorität aber auch die persönliche Reife und die Lebenserfahrung, die für viele mit dem Alter einhergeht, gemeint. Andere Unternehmensvertreter drücken aus, dass die Altersfrage ambivalent zu bewerten ist und das Alter nicht immer etwas über die Qualität des Coachs aussagt, beugen sich aber dennoch den Erwartungen der Coaching-Nehmer nach einem Gesprächspartner mit mindestens gleichem oder größerem Erfahrungshorizont. Weitere wichtige Faktoren sind die Haltung des Coachs, sein Rollen- und Menschenbild, die Methoden bzw. deren Vielfalt und indikationsgerechter Einsatz sowie die Passung zum Unternehmen. Diese definiert sich für die Unternehmensvertreter vor allem durch die Übereinstimmung in Werten, kulturellen Vorstellungen und der Sprache. Die mit der Sprache verbundene Kommunikationsfähigkeit wird eher als Hygiene-Kriterium gesehen, welches der Coach durch seine berufliche Ausrichtung grundsätzlich mitbringt. Coaches fokussieren darüber hinaus vor allem die Beziehungsfähigkeit, insbesondere Empathie und die Fähigkeit, Beziehung schnell aufzubauen. Diese stärkere inhaltliche Ausrichtung lässt sich mit der intensiveren Auseinandersetzung mit dem Gegenstand Coaching und dem Fokus auf die tatsächliche Arbeit mit dem Klienten, die für Unternehmensvertreter üblicherweise nicht einsehbar ist, begründen.

\subsection{Dienstleistungen zur Coach-Auswahl und ihre Bewertung}

Die vierte und letzte Forschungsfrage beschäftigt sich schließlich mit dem Angebot von Coach-Maklern und ihren Dienstleistungsunternehmen, Aufgaben und Leistungen, um die Coach-Auswahl und Organisation für Unternehmen zu übernehmen. Der Coaching-Markt allgemein wird sowohl von Unternehmensvertretern als auch Coaches mit einer quantitativ hohen Dichte und einer sehr heterogenen Qualität beschrieben, was nach Ansicht der Befragten in die Beschreibung eines schwer überschaubaren und intransparenten Marktes mündet.

Trotz dieser Einschätzung reagieren die befragten Unternehmensvertreter zu großen Teilen mit Ablehnung auf Coach-Makler, die den Markt stellvertretend strukturieren wollen. Zehn von 13 Unternehmen sehen keinen Bedarf oder Sinn in der Kooperation mit solchen Organisationen. Der von den Befragten genannte Grund dafür lautet, dass Coaching kein Massengeschäft sei, welches sich mit den internen Ressourcen gut abdecken lasse. Außerdem wird ein Wissens- und Qualitätsverlust in Prozessen, im Matching von Coach und Klient und in der Person des Coachs befürchtet. So sind zwei Unternehmensvertreter der Ansicht, dass auf den Plattformen und in den Pools solcher Coach-Makler nur Coaches gelistet seien, denen durch fehlendes Empfehlungsmanagement der Zugang zu Unternehmen verwehrt bleibe. Dies werten die Unternehmensvertreter als Indiz für die mangelhafte Qualität der Coaches. Auch die Coaches selbst sind vereinzelt dieser Ansicht. Um gute Qualität gewährleisten zu können, wollen Unternehmensvertreter diese Verantwortung und die entscheidende Aufgabe niemand anderem anvertrauen: „Wo ich jemanden auf, auf eine ganz wichtige Person lasse, nämlich auf meinen Kunden, der mir vertraut, und ich hätte nur über indirekte Kontakte Einfluss da drauf. Das würde ich nicht machen“ (Interview 18, Schilling 2019; S. 236). Auch wird in der selbstverantworteten Coach-Auswahl und der damit sichergestellten Qualität ein Signal für die Werte und Kultur des Unternehmens und darüber hinaus für die eigene Daseinsberechtigung gesehen: „Dann braucht man meine Rolle nicht" (Interview 16, Schilling 2019; S. 207). Auch wenn die Nutzung solcher Dienstleistungsangebote von fast allen Unternehmensvertretern für den eigenen Verantwortungsbereich abgelehnt wird, sind für die Befragten dennoch An- 
wendungsgebiete denkbar. Aus ihrer Sicht hilfreich können solche Angebote für kleine Unternehmen ohne Personalentwicklung und mit wenig Ressourcen sowie bei häufigen Standard-Anfragen sein.

Auch die befragten Coaches zeigen sich den Maklerangeboten und -plattformen eher verhalten gegenüber. Zwei der befragten Coaches sind bei größeren Online-Plattformen gelistet, haben bisher aber keine Aufträge darüber generiert. Die Aufnahme geschah in einem Fall über die Zertifizierung eines anderen, zwischengeschalteten Kooperationspartners und im anderen Fall über die Direktansprache des Coachs. Es wurde kein weiteres Aufnahmeprozedere durchgeführt. Wie das Matching erfolgt und welche Anforderungen an die Coaches zur Aufnahme gestellt werden, ist für die Coaches nicht ersichtlich. Aus Sicht der befragten Coaches besteht aufgrund der eigenen Auslastung keine Notwendigkeit, diese Dienstleistungen in Anspruch zu nehmen. Neben der Gefahr, monetäre Einbußen durch Provision zu erleiden, sehen Coaches wie auch Unternehmensvertreter das Risiko, mit dem Einbezug von Kooperationspartnern interne Signale zu senden und wichtige interne Prozesse zu verlieren: „Organisationen sollen sich bitte nicht outsourcen! Sondern sie sollen sich damit auseinandersetzen. Organisationen sollen sich klar machen, was sie eigentlich brauchen, was sie wollen für ihre Mitarbeiter. Was es auch für jemand sein muss, der auch irgendwie die Organisationskultur vielleicht mit vertritt oder aufnimmt. Ich fände das schade, wenn das verloren geht, wenn das nach draußen verlagert wird. Da geht was ganz Wichtiges vom Coaching verloren“ (Interview 19, Schilling 2019; S. $246 \mathrm{ff}$.).

Diesen kritischen Haltungen gegenüber stehen die Aussagen des Interviewpartners, welcher der Unterstichprobe der Coach-Makler zuzuordnen ist. Zunächst wird, wie bereits erläutert, kritisiert, dass die Verantwortung der CoachAuswahl oft zentral in der Personalabteilung verankert ist, nicht jedoch bei den eigentlichen Endkunden, den Coaching-Nehmern. Die Dienstleistung dieses Interviewpartners besteht jedoch nicht allein in der Bereitstellung eines Portals, sondern vor allem in der ganzheitlichen Konzeption eines Coaching-Ansatzes spezifisch für das Unternehmen. Die Coaches werden in diesem Zuge vom Befragten durch eigene Recherche und Empfehlungen selbst ausgewählt und in einem nicht-strukturierten Auswahlgespräch und einer sich anschließenden kurzen Simulation geprüft. Als Vorteile der Dienstleistung werden vor allem die Kostenreduktion und mehr Diversität und Fluktuation im Coach-Pool gesehen. Der Interviewpartner konstatiert schließlich eine Marktentwicklung, die Coaching zu einem Massenprodukt ohne Rücksicht auf Qualitätsverluste machen will bzw. bereits gemacht hat. Die einzige Art und Weise, diesem Markt zu begegnen, sei die Zusammenarbeit mit einem Partner. Als mögliches Zielszenario sieht der Dienstleistungsvertreter eine intelligente Suchmaschine, die zur Suche von Coa- ches genutzt werden kann, die aber für niemand eine monetäre Einnahmequelle ist. Abschließend sieht der Dienstleistungsvertreter weitere Potenziale zur Professionalisierung des Marktes und der Dienstleistung Coaching nicht in weiteren Regularien und Strukturierungen des Marktes durch externe Kräfte, sondern im eigenen Professionalisierungsparadigma. Dies bedeutet, dass sich Coaches die eigene Weiterentwicklung sowie Professionalisierung ihrer Tätigkeit als Ziel und Maßgabe selbst auferlegen und in ihrer täglichen Arbeit stets berücksichtigen müssen.

\section{Diskussion}

\subsection{Anforderungsanalyse - ein impliziter Passepartout}

Personalauswahl beginnt mit dem Erstellen eines Anforderungsprofils. Eine professionelle Anforderungsanalyse wird in den Unternehmen in der Regel nicht durchgeführt. Als Grund für die fehlende Anforderungsanalyse wird oft die Einzigartigkeit jedes Prozesses genannt, welcher auch die individuelle Entwicklung von Anforderungsprofilen erfordert. Der Aufwand dafür sei zu hoch, daher unterbleibe er. Doch damit wird die Auswahl willkürlich. Die Definition einer Passung wird zudem in den individuellen Matchingprozess von Coach und Klient verlagert, was diesen tendenziell überfrachtet, da eine nennenswerte Vorauswahl nicht stattgefunden hat. Anzunehmen ist außerdem, dass durch diese Verlagerung Verantwortungsdiffusion entsteht und Rollen, Verantwortlichkeiten und Professionalisierung der Personalentwicklung weiter verwischen.

Kriterien, die bei Fragen nach den Anforderungen genannt werden, sind noch unspezifisch und anfällig für implizite Theorien (Lebens-, Berufs-, Coaching-Erfahrung). Sie stellen eher Kompetenzvermutungen dar. So wird vielfach von Coaches Führungserfahrung gefordert. Laut Kanning und Fricke (2013) zeigen Personen ohne Führungserfahrung jedoch geringfügig höhere Werte auf der Dimension Führungskompetenz als die angeblich Erfahrenen. Unterschlagen wird mit der formulierten Anforderung zudem, dass nichtleitende Mitarbeiter ebenfalls über relevante Erfahrung verfügen. In einen anderen Kontext gesetzt, würde so gefordert, dass nur Suchttherapie durchführen dürfte, wer selbst auf eine Drogenkarriere zurückblicken kann. Die Absurdität eines solchen Anforderungsprofils gipfelt in der - in der Personalpsychologie wohlbekannten - Metapher „Schmidt sucht Schmidtchen“. Ein Ähnlichkeitsmodell, das einerseits mit Sorge um Anschlussfähigkeit übersetzt werden kann, andererseits auch mit „Wasch' mir den Pelz, aber mach mich nicht nass“. Das Ähnlichkeitsmodell ist eine voraussetzungsreiche, zumeist implizite Vorannahme. Bei etlichen Fragestellungen hat sie sich als unzutreffend erwie- 
sen. So spielt eine Passung der Persönlichkeit von Coach und Klient nachweislich keine Rolle (De Haan et al. 2016).

Besonders bemerkenswert erscheint die Aussage der Personalentwickler, dass man einen Nachweis für eine abgeschlossene Coaching-Weiterbildung vom Coach einfordert, was grundsätzlich als ein guter Ansatz erscheint. Die Ausbildungsangebote auf dem Markt sind allerdings sehr heterogen, sodass ein solches Kriterium mutmaßlich keinerlei Aussagekraft besitzen kann. Sinnvoll wäre es daher, zudem eine Verbandszugehörigkeit zu fordern, denn Verbände setzen Standards und involvieren ihre Mitglieder in kontinuierliche Weiterbildung und Austauschprozesse. Dass den befragten Einkäufern die Verbandszugehörigkeit so wenig wichtig ist, zeigte auch schon die erste Marburger Coaching-Marktstudie (Gross und Stephan 2015) und zuletzt wieder Finke und Kanning (2019). Als Hypothese ließe sich formulieren, Personalentwickler betrachten CoachingVerbände als inhaltliche Konkurrenten, sie fürchten, die Deutungshoheit aus der Hand zu geben. Bemerkenswert ist in diesem Zusammenhang der geringe Anteil an Unternehmensvertretern, die bspw. im Deutschen Bundesverband Coaching (DBVC) organisiert sind (19 von 467 Mitgliedern; Stand: 2019) - wobei dieser Verband der einzige ist, der dezidiert die Mitgliedschaft von Unternehmensvertretern propagiert. Auch die Stiftung Warentest (2014) hat einen Impuls zu Mindeststandards von Coach-Weiterbildungen gegeben, dieser konnte bislang offenbar keine nachhaltigen Konsequenzen in der Praxis der Verbände zeitigen. Als gravierendes Hindernis für eine Orientierung muss registriert werden, dass Coaching als Weiterbildung gilt, die eine Grundqualifizierung (Beruf) voraussetzt. Daher wird die Tätigkeit prinzipiell nicht durch ein Berufsrecht reguliert. Eine europaweite Erhebung konstatiert daher, „dass SupervisorInnen \& Coaches in einem mehrheitlich gesetzlich nicht geregelten Gewerbe tätig sind“ (Anger und Schober 2014, S. 50).

Zusammenfassend kann festgehalten werden, eine professionelle Anforderungsanalyse wird in den Unternehmen noch wenig durchgeführt, ein klares Anforderungsprofil für die Coach-Suche und -Auswahl liegt in der Regel selten vor. Als Lösung hierfür ließen sich Anforderungskriterien an Coaches vom Konzept der Wirkfaktoren (Behrendt und Greif 2018) ableiten. Die Anforderungsanalyse ließe sich mit wenig Aufwand substanziell verbessern. Es können spezifische Wissensbestände benannt oder eingegrenzt werden. Einschränkend äußern diese Autoren jedoch, dass Wirkfaktorenmodelle wichtige Anhaltspunkte liefern, in ihrer Gesamtheit aber noch zu wenig empirisch untersucht sind. Gleiches gilt für die Alternative, inzwischen vorliegende Kompetenzkataloge (Rauen und Steinke 2019) zu nutzen.

\subsection{Der Coach-Einkauf - ein Closed Shop}

Auch über zehn Jahre nach der Untersuchung von Kühl (2006) präsentiert sich der Coach-Einkauf sehr stark als Empfehlungsgeschäft. Dass ein weiterer Weg der Geschäftsanbahnung über den Ausbau einer bestehenden Zusammenarbeit (bspw. als Trainer) verläuft, unterstützt diese These weiter. Zudem werden der Wille bzw. die Notwendigkeit für Veränderung durch die Aussagen der Interviewten wenig sichtbar. Die Unternehmen sehen keine Priorität, das Prozedere zu verändern. Coach-Pools erscheinen damit tendenziell als Closed Shop.

Dies ist verwunderlich, werden doch jährlich alleine von den über 400 beim Portal coaching-index.de gelisteten Coaching-Weiterbildungsinstituten unzählige Coaches auf den Markt entlassen. Was passiert mit diesen Coaches? Wie gelangen sie an Aufträge? Zur Erklärung ließen sich folgende Hypothesen generieren, die die weitere Forschung anregen können: Neben der Kompetenzentwicklung, einer Zertifizierung und dem damit verbundenen potenziell erleichterten Marktzugang als Coach müssen weitere starke alternative Motive für die Teilnahme an einer CoachingWeiterbildung vorliegen. Dies können bspw. der Anschluss an soziale Netzwerke, eine generalisierende Kompetenzentwicklung für die Rolle als Mitarbeiter oder Führungskraft sowie ein erhoffter persönlicher Image-Gewinn im Sinne einer Aufwertungsstrategie (Upgrading) sein.

Die Möglichkeit, im Markt auf unseriöse Anbieter zu stoßen, wird von den Interviewpartnern grundsätzlich wahrgenommen. Die von Dritten erhaltenen Empfehlungen fungieren daher als Filter, können aber ebenso anfällig für Wahrnehmungsfehler und Verzerrungen sein. Die Sicherheit der befragten Unternehmensvertreter resultiert häufig aus der von ihnen empfundenen Bewährtheit des Systems, denn Beschwerden oder misslungene Matches gäbe es kaum oder gar nicht. Nachrichten über misslungene Coaching-Prozesse, so die Hypothese, dringen vermutlich deshalb nicht an die Oberfläche, weil die Prozesse nicht oder lediglich oberflächlich evaluiert werden. Auch im Falle eines mit objektiver Evaluation erfassbaren Problems im Coaching-Prozess lassen sich darüber hinaus auch gute Gründe dafür finden, dass Klienten ihre Unzufriedenheit der Personalentwicklung gegenüber nicht aktiv äußern. Schon Kühl (2006) wies darauf hin. Die Aura „unter vier Augen“ (Refugium) dient daher nicht nur dem Schutz des Klienten gegenüber seinen Vorgesetzten oder der Personalabteilung, sondern die Vertraulichkeit bietet auch Coaches vielfältige Freiheitsgrade. Weder Personalentwicklung noch Klienten sind in der Regel Coaching-Experten, kritisches Feedback kann mit einer autoritären Geste des Coachs leicht relativiert werden. Kritische Äußerungen seitens des Klienten macht diesen selbst angreifbar, da er dann seinerseits über sensible Dinge berichten muss, derentwegen er nun gerade den vertraulichen 
Coaching-Rahmen gesucht hat. Der geschützte Rahmen im Coaching ist folglich eine ambivalente Angelegenheit, der neben der Evaluation auch die Auswahl und regelmäßige Überprüfung des Coach-Pools erschwert. Folglich bestätigt sich die von Stephan und Gross (2011) angeführte Schwierigkeit im Umgang mit personenbezogenen Dienstleistungen: Deren Qualitätsüberprüfung ist in keinster Weise trivial. So bleibt der Einkauf zwangsläufig ein Erfahrungs- und Empfehlungsgeschäft.

Insofern erscheint das Verhalten der Coach-Einkäufer wiederum rational und theoriekonform: Empfehlungsmarketing überbrückt die Unsicherheit, hierauf verweisen schon Schoorman et al. (2007): Vertrauen ist ein Aspekt von Beziehungen. Insofern können Einkäufer, statt prinzipiell mit Skepsis zu reagieren, wie dies die von Gross und Stephan (2011) referenzierte Agency Theory erwarten lässt, ebenso gut begründet dem Coach einen Vertrauensvorschuss gewähren, wie dies die konkurrierende Stewardship Theory postuliert.

Unklar bleibt schließlich, wie viel Geschäft im Closed Shop stattfindet. Es bleibt offen, wie sehr der Coach-Pool aktiv eingesetzt wird oder ob er nicht gleichfalls eine AlibiFunktion erfüllt. Da Coaching eine teure Dienstleistung ist, könnte das tatsächliche Coaching-Volumen deutlich geringer ausfallen als landläufig behauptet wird. Die optimistische Aussage „Coaching boomt!“ kann allerdings von beiden Marktseiten in der eigenen Öffentlichkeitsarbeit imageförderlich genutzt werden: Die Unternehmen erscheinen modern, was im Zuge des Employer Branding attraktiv ist. Die Coaches erscheinen als gefragt und erfahren.

\subsection{Der Coach-Auswahlprozess - unbewusste Selbstsabotage}

Die Auswahl von Coaches in Unternehmen beschränkt sich überwiegend auf Auswahlgespräche, sehr vereinzelt werden umfangreichere Verfahren mit verschiedenem Methodenzugang durchgeführt. So decken sich die Ergebnisse dieser Studie mit den Resultaten vorangegangener quantitativer Befragungen (Gross und Stephan 2015; Finke und Kanning 2019). Auf eine Simulation wird oftmals aus Gründen der Künstlichkeit verzichtet. Fraglich erscheint an dieser Stelle, wie die Beziehungsfähigkeit und die Coaching-Kompetenz ohne eine solche Simulation überprüft werden kann. Mit geübtem Impression Management ist es denkbar, dass auch weniger kompetente Coaches die Auswahlgespräche erfolgreich durchlaufen. Es ist aufgrund der Aussagen der Befragten anzunehmen, dass die Gütekriterien eignungsdiagnostischer Verfahren in vielen Fällen verletzt werden. Die Nennung von Bauchgefühl, Intuition und Erfahrung als Treiber für die Entscheidung - auch im Sinne des Empfehlungsmanagements (Schoorman et al. 2007) - stellt bspw. die Objektivität der Verfahren deutlich in Frage. Durch die geringe Standardisierung ist ebenso die Durchführungsobjektivität verletzt. Auch die Konstruktvalidität, also diskriminante und konvergente Validität, wird schätzungsweise nicht erreicht, weil eine Überprüfung der Einschätzung über verschiedene methodische Zugänge meist fehlt.

Das Optimierungspotenzial des diagnostischen Anspruchs, welches in den Äußerungen von Einkäufern deutlich wird, steht klar im Widerspruch zur Relevanz, die der Coach-Auswahl seitens der Befragten beigemessen wird. Schließlich beschreiben alle Interviewten einstimmig den Coaching-Markt als unübersichtlich. Trotz dieser Wahrnehmung gestaltet sich die Auswahl nicht entsprechend eines solchen Chaos. Bei einem Verzicht auf eine anspruchsvolle Diagnostik wird die Verantwortung leichtfertig an den Markt delegiert. Viele der Unternehmensvertreter sehen nicht bei sich die Verantwortung, mit den Marktverhältnissen strukturiert umzugehen, sondern verlangen Veränderung, Organisation und Fortschritt durch externe Kräfte, die auf den Markt einwirken. Dies könnte als eine bequeme Forderungshaltung beurteilt werden. Ebenso ist das geringe Engagement nicht mit den Aussagen der Befragten konsistent, dass die Coach-Auswahl eine hohe Bedeutung für die eigene Rolle als Vermittler hat und laut den Befragten ein $\mathrm{Ma} ß$ für die eigene Kompetenz sei. Die Verantwortlichen möchten nach eigenen Aussagen außerdem den Grundstein für einen erfolgreichen Coaching-Prozess legen, da eine solche Maßnahme teuer, eher selten und von hoher Bedeutung für den Klienten ist. Das Potenzial zur Verbesserung des Auswahlprozesses nach DIN 33430 liegt vor und wäre mit überschaubarem Aufwand in hohem Maße zu realisieren. Bislang nutzen dies aber offenbar nur wenige Unternehmen und sabotieren sich unbewusst damit möglicherweise selbst.

\subsection{Dienstleistungen der Coach-Auswahl - eine Gefahr für Personaler?}

Die Nutzung und Relevanz von Dienstleistungen rund um die Coach-Auswahl werden von den aktuell Befragten durchgängig kritisch betrachtet. Eine mögliche Interpretation dieser Tatsache liefert die Hypothese, dass Dienstleistungsangebote in diesem Bereich die Daseinsberechtigung und die Verantwortlichkeit der entsprechenden betrieblichen Einkäufer gefährden, die Abwehrhaltung könnte der Selbsterhaltung dienen. Dies wiederum steht im Einklang mit den Ergebnissen von Kühl (2008a), der in der Kanalisierung von Coaching im Bereich Personalentwicklung vor allem eine Steigerung der Relevanz dieses Bereichs im Unternehmen sieht. Die Abwertung der Qualität externer Angebote würde somit der eigenen Aufwertung dienen. Auch die Kritik des Interviewpartners aus der Unterstichprobe der Dienstleistungsanbieter an der Verankerung des 
Tab. 2 Verbesserungsansätze und konkrete Umsetzungsvorschläge für die Optimierung von Coach-Auswahlverfahren zur gesteigerten Vereinbarkeit mit der DIN 33430

\begin{tabular}{|c|c|c|c|}
\hline & Verbesserungsvorschlag & Konkrete Umsetzung & Vorteil \\
\hline \multirow[t]{2}{*}{$\begin{array}{l}\text { Organisa- } \\
\text { tionsqualität }\end{array}$} & $\begin{array}{l}\text { Trimodaler Ansatz: Be- } \\
\text { leuchtung von Eigen- } \\
\text { schaften, Verhalten, Bio- } \\
\text { grafie (Schuler 2014) }\end{array}$ & $\begin{array}{l}\text { Ergänzung der Selbstpräsentation und biografischen } \\
\text { Fragen durch Verhaltensmessung in der Simulation } \\
\text { (z.B. Auftragsklärungsgespräch oder Coaching-Se- } \\
\text { quenz mit definiertem oder frei wählbarem Anliegen) }\end{array}$ & $\begin{array}{l}\text { Facettenreichste und vollständigste Be- } \\
\text { trachtung des Coachs, Steigerung der } \\
\text { Güte und Aussagekraft des Auswahlpro- } \\
\text { zesses }\end{array}$ \\
\hline & $\begin{array}{l}\text { Multimethodischer } \mathrm{Zu}- \\
\text { gang, Mehrfachbeobach- } \\
\text { tung und Beurteilung }\end{array}$ & $\begin{array}{l}\text { Neben Interviews auch Präsentation und Diskussion } \\
\text { sowie Simulationen oder Tests nutzen }\end{array}$ & $\begin{array}{l}\text { Verbesserung der diskriminanten und } \\
\text { konvergenten Validität, Verifizierung der } \\
\text { Einschätzung durch mehrere Beobach- } \\
\text { tungspunkte }\end{array}$ \\
\hline \multirow[t]{4}{*}{$\begin{array}{l}\text { Produkt- } \\
\text { qualität }\end{array}$} & $\begin{array}{l}\text { Critical Incidents Metho- } \\
\text { de (Flanagan 1954) }\end{array}$ & $\begin{array}{l}\text { Konstruktion spezifischer Fallgeschichten, bspw. zum } \\
\text { Thema Coaching in einer innerbetrieblichen Konfliktsi- } \\
\text { tuation oder Coaching in der Post-Merger-Integration }\end{array}$ & $\begin{array}{l}\text { Operationalisierung unspezifisch be- } \\
\text { nannter Anforderungen, Individualisie- } \\
\text { rung auf die Anforderungen des Unter- } \\
\text { nehmens/Klienten }\end{array}$ \\
\hline & $\begin{array}{l}\text { Multimodales Interview } \\
\text { (Schuler 2014) }\end{array}$ & $\begin{array}{l}\text { Entwicklung eines multimodalen Leitfadens (Ge- } \\
\text { sprächseinstieg, Selbstvorstellung, freies Gespräch, } \\
\text { Handlungswissen, Biografiebezogene Fragen, Tätig- } \\
\text { keitsinformation, situative Fragen, Gesprächsabschluss) }\end{array}$ & $\begin{array}{l}\text { Höherer Standardisierungsgrad, Steige- } \\
\text { rung der Vergleichbarkeit, Erleichterung } \\
\text { der Vorbereitung und Durchführung } \\
\text { (auch für neue Verantwortliche) }\end{array}$ \\
\hline & $\begin{array}{l}\text { Beurteilung durch Erwar- } \\
\text { tungshorizont }\end{array}$ & $\begin{array}{l}\text { Entwicklung eines Beurteilungsbogens mit numeri- } \\
\text { schem Beurteilungssystem, Operationalisierung durch } \\
\text { mögliche und geforderte Lösungsideen/Antworten und } \\
\text { deren Rating }\end{array}$ & $\begin{array}{l}\text { Standardisierung und validere Aussage- } \\
\text { kraft, Verbesserung der Vergleichbarkeit, } \\
\text { Steigerung der Güte und Aussagekraft } \\
\text { des Auswahlprozesses }\end{array}$ \\
\hline & $\begin{array}{l}\text { Feedback nach Abschluss } \\
\text { des Verfahrens }\end{array}$ & $\begin{array}{l}\text { Einholen der Wahrnehmung des Coaches, anschließend } \\
\text { wertschätzendes und offenes Feedback, Hinweise auf } \\
\text { Entwicklungsfelder }\end{array}$ & $\begin{array}{l}\text { Steigerung der Akzeptanz, weiterer An- } \\
\text { haltspunkt für die Reflexionsfähigkeit } \\
\text { des Coaches (ergänzend zu Fragen nach } \\
\text { Supervision und Weiterbildung) }\end{array}$ \\
\hline $\begin{array}{l}\text { Personen- } \\
\text { qualität }\end{array}$ & $\begin{array}{l}\text { Beobachtung und Beurtei- } \\
\text { lung durch Coaching-Ex- } \\
\text { perten }\end{array}$ & $\begin{array}{l}\text { Durchführung durch Personen mit eigener Coaching- } \\
\text { Ausbildung und Erfahrung (bspw. Personalentwick- } \\
\text { lung) }\end{array}$ & $\begin{array}{l}\text { Erhöhung der Personenqualität, Steige- } \\
\text { rung der Aussagekraft }\end{array}$ \\
\hline
\end{tabular}

Coachings in der Personalabteilung statt beim End-Nutzer steht im Einklang mit dieser Deutung.

Damit offenbart sich jedoch ein Widerspruch: Wenn die Coach-Auswahl derart kulturstiftend wirkt und das Menschenbild, die Personalpolitik und die Unternehmenskultur dadurch positiv unterstützt wird, warum zeigt sich dies nicht in einem gesteigerten Engagement und in einer höheren Priorität der Coach-Auswahl in den Unternehmen? Es muss folglich eine positive Funktion des Outsourcings bestehen, die bislang noch kaum in den Blick geraten ist.

Der Rückgriff auf das von Kühl (2008b) benannte Evaluationsdilemma kann das Outsourcing der Coach-Auswahl trotzdem funktional erscheinen lassen. Die betrieblichen Einkäufer müssen sich gegenüber den Linienmanagern, aber auch gegenüber der Geschäftsführung rechtfertigen. Da die Evaluation von Coaching aber ein sehr schwieriges Unterfangen ist (Vertraulichkeit, Individualität, Koproduktion etc.) und die eigenen Coach-Auswahlanstrengungen in der Regel weniger umfangreich sind, bietet das Outsourcing einen erheblichen Vorteil im Evaluationsdilemma: Die unangenehme Legitimationsfunktion wird outgesourct, Fehler liegen so beim externen Dienstleistungsanbieter. Man selbst kann sich auf die positive Funktion des Verbesserungsmanagements konzentrieren. Die von den Befragten geäußerte hohe Aufmerksamkeit für die Qualität des Coachings ist kompatibel mit dieser Funktion. Die unvereinbaren Rollen, mit denen die Personalentwicklung konfrontiert sind, werden durch das Outsourcing separiert (intern/extern). Eine damit verbundene Wertung der Arbeitsteilung entlastet die betriebliche Seite, die externe Seite lässt sich die Übernahme des Risikos angemessen hoch vergüten. Ein Geschäft zulasten eines Dritten: der Geschäftsführung, die den entsprechenden Mehraufwand finanzieren muss.

Einfacher stellt sich die Lage dar, wenn diese Dienstleistungsunternehmen kleine und mittelständische Unternehmen, die keine eigene Personalentwicklungsabteilung unterhalten, oder private Klienten ansprechen. Die SourcingIdee erscheint hier noch funktionaler. Für beide Szenarien ließe sich die Hypothese aufstellen, dass der Trend zum Outsourcen der Coach-Auswahl zukünftig stark anwachsen dürfte.

\subsection{Entscheidungen - einfache Verbesserungsvorschläge}

Auch wenn aus Unternehmens- und Coach-Sicht die Optimierungspotenziale für die Coach-Auswahl gering sind, sind dennoch aus externer Perspektive weitere Verbesserungsbedarfe erkennbar, die der Organisations-, Produkt- 
und Personenqualität zugeordnet werden können. In Tab. 2 sind einige dieser Vorschläge auf- und näher ausgeführt.

Weitere Anregungen liefern Veröffentlichungen zum Management-Audit (Weinert und Stulle 2015), denn Verfahren, die zur Auswahl von Managern eingesetzt werden, können ebenfalls für die Auswahl von Coaches genutzt werden.

Zusammenfassend muss konstatiert werden, dass der bisherige Auswahlprozess von Coaches der Qualitätssicherungsfunktion sowie den Anforderungen der DIN 33430 selten gerecht werden kann. Die schon von Kühl (2006) vorgetragenen Kritikpunkte lassen sich auch eine Dekade später nahezu deckungsgleich finden. Dies ist überraschend, weil zugleich immer wieder betont wird, wie wichtig es sei, geeignete Coaches zu finden und unpassende vom Unternehmen fernzuhalten. Ob dieses Vorgehen aus einer unwissenden oder einer willentlichen bis taktischen Haltung resultiert, ist offen.

\section{Kritische Würdigung}

Die vorliegende Studie unterliegt verschiedenen methodischen und inhaltlichen Einschränkungen. Die anfallende Stichprobe und die Akquisition der Interviewpartner bedingt vermutlich Selektionseffekte, vor allem in der Unterstichprobe der Coaches. Ein höherer Strukturierungsgrad der Interviews könnte zu einer erhöhten Vergleichbarkeit der Ergebnisse führen. Darüber hinaus wird die Erhebungssituation durch die telefonische Durchführung der Interviews nicht ausreichend kontrolliert. Der Einfluss von Störvariablen kann somit nicht abgeschätzt werden. Gegebenenfalls führt aber die Durchführung der Interviews via Telefon aufgrund der terminlichen Unabhängigkeit und des geringen Aufwands zu einer erhöhten Teilnahmebereitschaft. Die telefonische Durchführung kann außerdem durch die räumliche Distanz zu einer erhöhten Offenheit und Ehrlichkeit der Interviewpartner beitragen. Diese Fragen bleiben letztlich offen.

Das Datenmaterial ist lediglich durch eine Person kodiert und ausgewertet worden. Die Ergebnisse können subjektiv verzerrt sein, eine Zweitkodierung hätte das Codesystem sowie dessen Reliabilität und Validität verbessern können. Ein weiterer Kritikpunkt besteht in der fehlenden kommunikativen Validierung der Ergebnisse mit den Befragten.

Inhaltlich ist zunächst kritisch anzumerken, dass die dritte Unterstichprobe der Dienstleistungsanbieter mit nur einem Interviewpartner eindeutig unterbesetzt ist. Der Vergleich der drei Perspektiven ist somit nicht gewährleistet. Da es allerdings schlicht zum Geschäftsmodell der Makler gehört, ihre Coaches - ob fundiert oder nicht - auszuwählen, und sich dabei nach Möglichkeit nicht angreifbar zu machen, erscheint deren Zurückhaltung verständlich. Sie wollen das eigene Vorgehen in der Coach-Auswahl ungern transparent machen.

Des Weiteren ist es zweifellos inhaltlich interessant zu erfahren, inwieweit Eignungsdiagnostik und Auswahlprozesse in der Personalauswahl im Unternehmen insgesamt gestaltet sind. Aus untersuchungsökonomischen Gründen musste auf diese Erhebung verzichtet werden. Weitere Forschung ist daher notwendig.

Zunächst kann als weiteres Forschungsvorhaben die quantitative Überprüfung der Ergebnisse gesehen werden. Im konkreten Kontext des Forschungsgegenstands dieser Studie, den Prozessen zur Auswahl von Coaches, steht aber auch vor allem die Frage im Raum, inwiefern ein verändertes Vorgehen in der Auswahl wirklich zu einem qualitativ besseren und valideren Ergebnis führt. Der Beweis steht noch aus, dass ein Vorgehen nach eignungsdiagnostischer Norm ein aussagekräftigeres Ergebnis erzeugt. Die Annahme dafür ist nachvollziehbar und begründbar, dennoch ist es interessant, die Ergebnisse verschiedener Verfahren miteinander zu vergleichen oder die Ergebnisse verschiedener Auswahlprozesse und Beurteilungen mit kritischen Erfolgskriterien der Coach-Tätigkeit in Zusammenhang zu bringen.

Im Laufe der Analyse sind außerdem immer wieder Hinweise aufgetreten, dass der Status quo der Auswahl von Coaches auch mit dem Reifegrad des Unternehmens hinsichtlich des Coachings und der Personalentwicklung insgesamt zusammenhängen mag. Beispielsweise kann vermutet werden, dass die Implementierung von Coaching im Unternehmen und damit auch die Auswahlprozesse beispielsweise mit dem Image von Coaching, der Historie, der Verbreitung der Maßnahme, der Unternehmensgröße und vielen weiteren Faktoren zusammenhängt. Eine nähere Betrachtung der Faktoren, die die Implementierung von Coaching im Unternehmen beeinflussen, würde es ermöglichen, gezielt Ansatzpunkte für die Professionalisierung von Coaching im Unternehmen zu identifizieren und diese voranzutreiben.

\section{Fazit}

Coaching im Unternehmen kann auf Grundlage der erhobenen Daten als Closed Shop gesehen werden. Man bleibt im Empfehlungsgeschäft unter sich. Beliebtestes Auswahlinstrument in der vorliegenden Stichprobe ist das Auswahlgespräch. Die Anforderungen, die an Coaches gestellt werden, bewegen sich zurzeit noch stark auf der Ebene der Kompetenzvermutungen, das Bauchgefühl und das entwickelte Vertrauen zum Coach sind für die Coaching-Verantwortlichen oftmals entscheidend. Dienstleistungsangebote werden insgesamt von den Befragten eher kritisch gesehen. 
Interessant sind vor allem die Widersprüche, die sich in der Untersuchung offenbaren: Der Markt wird als intransparent und Dienstleistungsangebote als unpassend für die interne Zielsetzung der verstärkten Mitarbeiterorientierung bewertet. Dennoch erfolgt noch keine diagnostisch anspruchsvolle Auswahl, obwohl die Maßnahme teuer und das Gelingen des Prozesses für den Klienten und die Position des Verantwortlichen besonders wichtig ist. Man gewinnt zudem den Eindruck, dass weder die Mehrheit der Coaches noch der Unternehmen ein großes Interesse daran haben, den Status quo zu verändern - aus welchen Gründen bleibt unklar und Gegenstand von Spekulationen.

Aus professioneller Perspektive erscheinen Optimierungspotenziale leicht zu realisieren. Schon Kühl (2006) äußerte die Vermutung, dass die Kompetenz dafür in den Unternehmen noch zu wenig ausgeprägt ist. Die Aus- und Weiterbildung der betrieblichen Einkäufer und die Orientierung an Best Practice (DIN 33430) wäre daher zu fordern. Etliche Jahre nach ihrer Veröffentlichung wird die Umsetzung dieser Norm noch als zu gering bezeichnet (Kersting 2009).

Es konnte darüber hinaus auch eine erhebliche, bislang unbeachtete Funktionalität der kritisierten Praxis entdeckt werden: Wenn Coaching eine professionalisierte Dienstleistung ist, die sich durch Immaterialität, Individualität, Koproduktion, Vertraulichkeit und Unabhängigkeit auszeichnet (Greif 2015), kann dies einige Personalentwickler einerseits überfordern. Andererseits eröffnet ihnen die Kapitulation vor der Aufgabe der professionellen Coach-Auswahl auch neue Freiheiten. Das Outsourcing der Aufgabe immunisiert ihre Funktion gegen Kritik. Es bleibt für Außenstehende allerdings weniger ersichtlich, ob die Kapitulation vor dieser Aufgabe und der Verzicht auf eine Professionalisierung der Auswahl eine bescheidene, weise Strategie darstellt angesichts der hohen Ansprüche und Schwierigkeiten einer Evaluierung des Coaching-Erfolgs oder schlicht naiv ist und verantwortungsloses Verhalten darstellt.

Nun spricht nichts gegen eine Doppelstrategie: Professionalisierung der Coach-Auswahl bei gleichzeitiger Bescheidenheit und Umsicht angesichts der hohen Ansprüche und Schwierigkeiten. Bei dieser Überlegung sollte sorgsam abgewogen werden, bis wohin die Kompetenzen zur eigenen Professionalisierung ausreichen und ab wann der Einbezug externer Expertise sinnvoll erscheint. Die CoachAuswahl lässt sich nach einem gängigen Konzept des Qualitätsmanagements (Heß und Roth 2001) als Strukturqualität bezeichnen (Input). Hier gute Arbeit zu leisten, produziert nicht die Garantie guter Ergebnisse. Allerdings erscheint es weniger wahrscheinlich, mit Defiziten beim Input eine gute Prozessqualität zu unterstützen. Insofern ist die Coach-Auswahl eine wichtige Voraussetzung für professionelle Coaching-Prozesse in Unternehmen. Daher sollte sie möglichst gewissenhaft betrieben werden.
Interessenkonflikt L. Schilling und T. Webers geben an, dass kein Interessenkonflikt besteht.

\section{Anhang}

\section{Idealmodell zur Coach-Auswahl - Modellbeschreibung}

Das im Folgenden beschriebene Modell bezieht sich ausschließlich auf die Auswahl von Coaches, die Unternehmen eigenständig vornehmen. Eine Auswahl, die durch einen Dienstleistungsanbieter erfolgt und die Auswahl von internen Coaches sind hiermit nicht abgedeckt. Das entwickelte Modell bezieht in seinem Aufbau verschiedene Vorgehensweisen der interviewten Unternehmen sowie die Verbesserungsvorschläge der Befragten mit ein. Das Modell wird darüber hinaus durch selbst abgeleitete Verbesserungsbedarfe und Aspekte der DIN 33430 (Westhoff et al. 2010) ergänzt.

\section{Überblick}

Das entwickelte Modell konzentriert sich nicht nur auf die reine Auswahl mithilfe von verschiedenen Instrumenten, sondern beginnt bei der Anforderungsanalyse. Außerdem geht das Modell über die Auswahlentscheidung hinaus und endet mit dem Feedback an den Coach. Grundsätzlich sollte der Auswahlprozess von mindestens zwei geschulten Beobachtern durchgeführt werden. Abb. 1 zeigt den Auswahlprozess und die dazugehörigen Schritte sowie Materialien und Dokumente.

Insgesamt nimmt dieses Modell die Standardisierung und Dokumentation der einzelnen Prozessschritte stärker in den Fokus und sorgt so für eine erhöhte Nachvollziehbarkeit, wie in der DIN 33430 gefordert (Westhoff et al. 2010). Dabei kann das Modell die einzelnen Prozessschritte und die beispielsweise dafür notwendigen Dokumente nicht vollumfänglich und mit hohem Detaillierungsgrad darstellen. Folglich kann auch ein nach DIN 33430 benötigtes Handbuch (Westhoff et al. 2010) für den entworfenen Auswahlprozess nicht vorgelegt werden. Im Folgenden sollen nun - unter Berücksichtigung dieser Einschränkungen - allerdings einige Aspekte des Modells speziell herausgegriffen und näher erläutert werden.

Anforderungsanalyse Den ersten Schritt stellt nach DINkonformem Vorgehen die Anforderungsanalyse dar (Reimann 2010). Im entwickelten Modell wird dazu ein teilstrukturiertes Verfahren, beispielsweise mit einem Interview oder der Critical Incident Technique (Flanagan 1954), vorgeschlagen. Auch die bereits bestehenden Anforderungen sind dabei zu berücksichtigen (Reimann 2010). Nach 
Abb. 1 Idealmodell zur CoachAuswahl. (Eigene Darstellung)

\section{Phase}

Inhalt

Dokumentation/Material

Anforderungs analyse

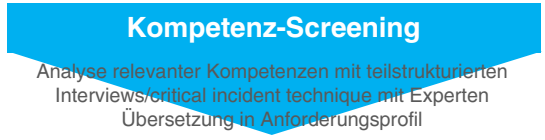

Anbahnung

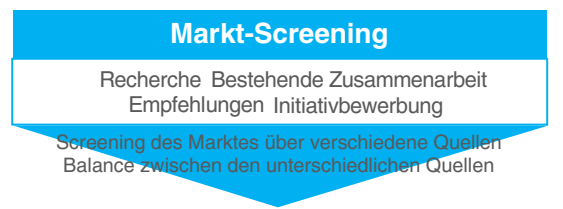

Vorauswah

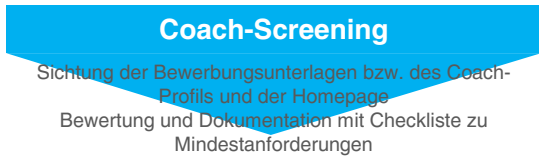

Erstkontakt

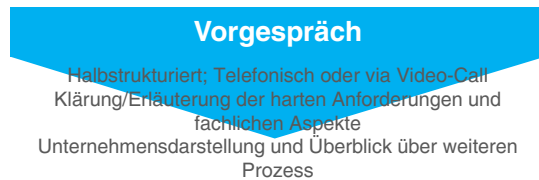

Auswahlverfahren
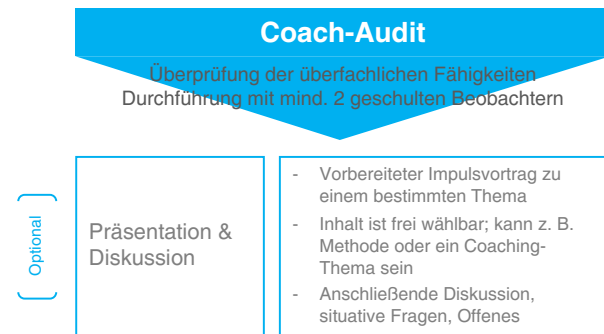

Vorbereiteter Impulsvortrag zu einem bestimmten Thema Inhalt ist frei wählbar; kann z. B. Methode oder ein CoachingThema sein

Anschließende Diskussion, Anschließende Diskussion,
situative Fragen, Offenes

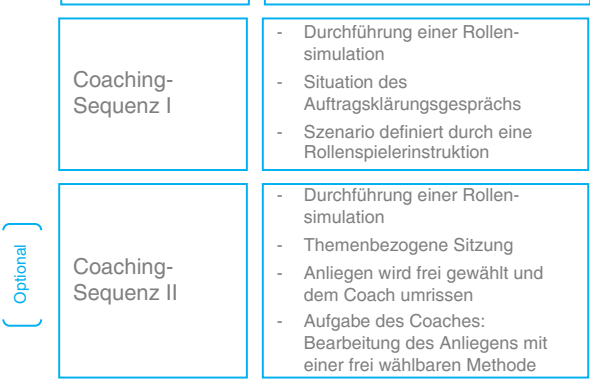

Auswahlverfahren

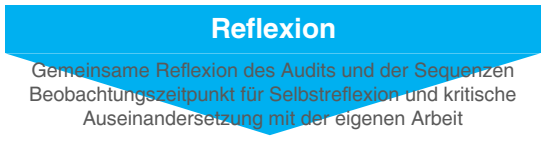

Entscheidung

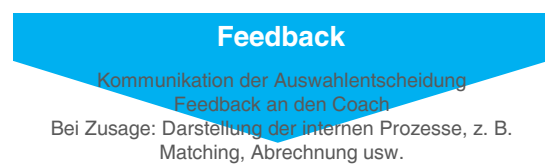

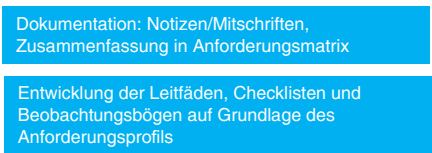
Anforderungsprofils
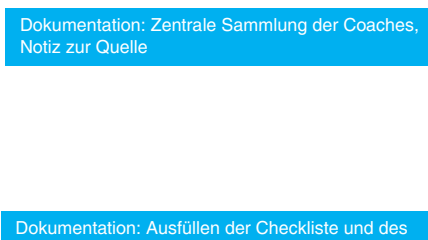
Profils mit den Eckdaten
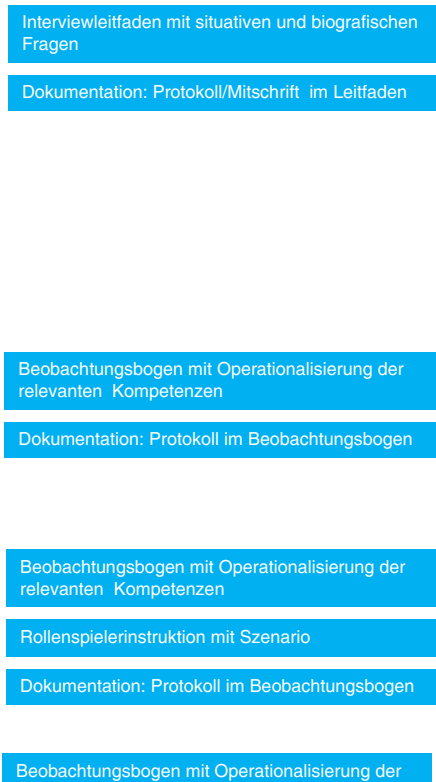

relevanten Kompetenzen

Dokumentation: Protokoll im Beobachtungsbogen
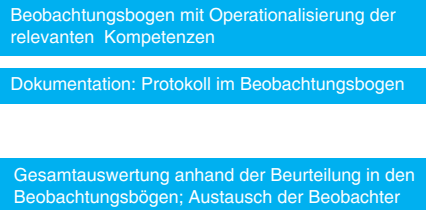

Kann im Anschluss an das Verfahren oder an einem anderen Tag durchgeführt werden

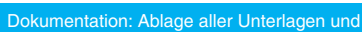

Dokumentation: Ablage aller Unterlagen und
Durchführung der Anforderungsanalyse entsteht das Anforderungsprofil des Coaches, den das Unternehmen sucht. Dieses dient als Grundlage für den Auswahlprozess und muss anschließend operationalisiert und in die einzelnen Dokumente übersetzt werden.
Anbahnung Der zweite Schritt des Modells spricht die zurzeit übermäßige Vorherrschaft des Empfehlungsgeschäfts an. Empfehlungen von Dritten sind sicher nichts vollkommen Schlechtes, dennoch muss sich in einem gesunden Markt, der gute Qualität und faire Wettbewerbsbedingungen bietet, der Zugang zu Unternehmen offener und trans- 
parenter gestalten. Daher sollte eine ausgeglichene CoachSuche über verschiedene Kanäle angestrebt werden. Einige Quellen sind im Modell aufgezeigt, diese können aber auch ergänzt werden.

Vorauswahl und Erstkontakt Die Vorauswahl, wie sie auch im Sinne der DIN 33430 vorgesehen ist (Westhoff et al. 2010), geschieht in den nächsten zwei Schritten. CoachScreening und Vorgespräch bilden die Inhalte ab, die aktuell in den Auswahlgesprächen thematisiert werden, und prüfen die harten Anforderungen ab, beispielsweise CoachErfahrung, Ausbildungen, Erfahrungshintergrund im Wirtschaftskontext etc. Diese Schritte sind dem Screening aus der Agency-Theorie zuzuordnen. Durch die Auskünfte im Gespräch und in den bereitgestellten Unterlagen erteilt der Coach Auskunft über sich (Signaling). Im Vorgespräch kommt jedoch auch das Signaling auf Unternehmensseite hinzu: Hier informiert das Unternehmen über die Zielgruppe des Coachings, Ablauf und Rahmenbedingungen des Coachings sowie über eventuell aktuelle Themen der Personal- und Organisationsentwicklung usw. Bei einer bedarfsspezifischen Auswahl wäre es an dieser Stelle möglich, den Coach über das Anliegen zu informieren. Die Informationspflicht des Unternehmens kommt außerdem im Übergang zum nächsten Schritt zum Tragen. Ist die Vorauswahl erfolgreich abgeschlossen und soll der Coach im Coach-Audit näher auf Eignung geprüft werden, so muss der Coach entsprechend der Norm (Westhoff et al. 2010) vollständig und transparent über das weitere Vorgehen und die Inhalte des Coach-Audits informiert werden.

Auswahlverfahren Das Coach-Audit fokussiert überwiegend überfachliche Kompetenzen des Coaches und entfernt sich von den Kompetenzvermutungen der aktuellen Auswahlverfahren. Die Präsentationsaufgabe dient zum Einstieg in das Audit und bietet Raum, um ggf. noch bestehende Fragen aus dem Vorgespräch zu klären und auf bisher noch unklare Aspekte im persönlichen Kontakt nochmals einzugehen. Die Aufgabenstellung der Präsentation kann ein vorgegebenes Thema umfassen, beispielsweise die Vorstellung einer Methode zur Entscheidungsfindung o. ä. Es wäre auch denkbar, den Coach dazu aufzufordern, seine Arbeitsweise und die Wirkmechanismen, die im Coaching greifen, zu präsentieren. Auch die Vorbereitung und anschließende Skizzierung eines Coaching-Falls wäre denkbar. Die anschließenden Coaching-Sequenzen stellen simulationsorientierte Verfahrenselemente dar. Die erste Sequenz simuliert die Situation der Auftragsklärung. Für eine höhere Objektivität und Vergleichbarkeit ist es an dieser Stelle sinnvoll, eine Instruktion für den Rollenspieler zu konzipieren, die das Szenario detailliert vorgibt. Herausforderung für den Coach könnte es sein, hinter das tatsächliche „Anliegen hinter dem Anliegen“ zu gelangen.
Beziehungsfähigkeit sowie Kommunikationsfähigkeit wären mögliche Kompetenzen, die zu beobachten wären. In der zweiten Sequenz soll eine Anliegen bezogene Interaktion zwischen Coach und Rollenspieler sichtbar werden. Für eine erhöhte Authentizität und stärkeren Anforderungsbezug wäre es denkbar, ein tatsächliches Anliegen des Rollenspielers zu nutzen. Das Anliegen soll dann in ersten Ansätzen mit dem Coach gemeinsam bearbeitet werden. Die drei exemplarisch dargestellten Übungen werden mithilfe eines Beobachtungsbogens zunächst beobachtet und anschließend systematisch bewertet. Das Coach-Audit ebenso wie der gesamte Prozess erfüllen so im Hinblick auf die DIN 33430 die geforderte Methodenvielfalt durch unterschiedliche Zugänge und Datenquellen (Kersting 2010).

Reflexion Den letzten Beobachtungszeitpunkt im CoachAudit stellt die gemeinsame Reflexion dar. Hier soll das Verhalten des Coaches während des Audits mit den Beobachtern reflektiert werden. So kann die Reflexionsfähigkeit des Coaches erfasst werden. Dies eröffnet die Möglichkeit, die selbstkritische Haltung und Auseinandersetzung des Coaches sowie die Weiterentwicklung seiner eigenen Arbeit real zu erfahren. Wie in den vorangegangenen Übungen wird auch in der Reflexion ein Beobachtungsbogen verwendet.

Feedback und Entscheidung Den Abschluss des CoachAudits sowie des gesamten Auswahlverfahrens bildet das Feedback an den Coach. Ein ausführliches und konstruktives Feedback steigert die Akzeptanz dieses Verfahrens und fördert die Interaktion zwischen Coach und Unternehmen. Dies ist wiederum eine Gelegenheit für Signaling auf Unternehmensseite: Coaches werden durch das Feedback wertgeschätzt und die individuelle Auseinandersetzung mit dem Coach zeigt den Stellenwert und die Bedeutung von Coaching im Unternehmen auf - auch für andere Stakeholder.

\section{Anmerkungen}

Schließlich ist es wichtig anzumerken, dass dieses Modell eine Anregung und erste Idee darstellt, wie Auswahlprozesse für Coaches gestaltet werden können. Es dient zum Aufbau und zur Erweiterung eines Coach-Pools oder kann auch für die Zurücksetzung eines Pools genutzt werden. Den Unternehmen, die bereits einen Pool an Coaches haben, ist zu empfehlen, diesen auf seine Qualität hin zu testen, wenn dies bisher noch nicht durch ein Auswahlverfahren sichergestellt wurde. Je nachdem, ob ein Coach für ein spezielles Thema oder Anliegen gesucht wird, ist es auch möglich, diesen Prozess in den ersten Auswahlschritten im Hinblick auf die harten Anforderungen hin anzupassen. Beispielsweise kann der Themenschwerpunkt des Coaches, welcher 
zum Anliegen des Klienten passen sollte, eine Mindestanforderung darstellen, die bereits im Coach-Screening überprüft wird.

Einige der Bestandteile sind modular zu verstehen und können je nach Bedarf verändert bzw. ausgelassen werden. Je mehr Prozessschritte und Beobachtungszeitpunkte jedoch geschaffen werden, desto aussagekräftiger und informativer ist die Auswahl bzw. die Auswahlentscheidung. Das Verfahren sollte von mindestens zwei Beobachtern durchgeführt werden. Die Beobachter sollten, wie in der DIN 33430 vorgesehen (Bühner 2010), im Vorfeld zum Gegenstand und zur Verfahrensweise sowie zu Beurteilungs- und Wahrnehmungsfehlern geschult werden.

Resümee Abschließend lässt sich festhalten, dass das Modell einen ganzheitlichen Ansatz darstellt und sich in seinen Grundzügen an der DIN 33430 orientiert. Insbesondere der vielfältige methodische Zugang, die systematische Beobachtung und Beurteilung anhand von operationalisierten Kompetenzen, die zuvor aus der Anforderungsanalyse abgeleitet wurden, und die Dokumentation unterstützen diese Ausrichtung. Dort, wo Inhalte von Übungen frei gewählt werden können, beispielsweise in der zweiten CoachingSequenz, und wo demnach keine Rollenspielerinstruktion vorliegt, werden die Standards nicht stringent eingehalten. Für eine vollständige Konformität mit den Richtlinien der DIN 33430 fehlen auch einige Aspekte oder bleiben bisher unberücksichtigt. So können erst eine vollständige und detaillierte Konzeption inklusive einer realen Anforderungsanalyse und die anschließende Evaluation des Vorgehens die Validität und damit die Qualität im Sinne der DIN 33430 endgültig bestätigen.

Einschränkungen Das abgeleitete Idealmodell kann als sehr umfangreich und für die Praxis nicht umsetzbar kritisiert werden - auch aus dem Grund, weil in der Umsetzung eignungsdiagnostische Kompetenzen vorausgesetzt werden, von denen nicht bekannt ist, in welchem Ausmaß sie im Einzelfall in den Unternehmen vorliegen. Wenn die Ableitung und Konzeption kritisiert wird, weil in der Praxis kein Bedarf erkannt wird, muss erwidert werden, dass Forschung immer die Aufgabe hat, vorzudenken und zu inspirieren. Allerdings darf auch die Forschungsseite diese Diskussion nicht verkürzen und sich rechthaberisch verweigern, wie dies leider beobachtet werden kann (Reimann et al. 2009a, 2009b). Wenn es nicht gelingt, den Horizont der eigenen Argumentation zu übersteigen (Klehe 2008) und in einen Dialog mit den Praktikern auf Augenhöhe zu treten, verkommt die Ableitung eines Idealmodells zu einer Übung im Elfenbeinturm. Das wäre schade und überflüssig. Denn nichts ist so praktisch - kolportieren wir hier Kurt Lewin - wie eine gute Idee!
Open Access Dieser Artikel wird unter der Creative Commons Namensnennung 4.0 International Lizenz veröffentlicht, welche die Nutzung, Vervielfältigung, Bearbeitung, Verbreitung und Wiedergabe in jeglichem Medium und Format erlaubt, sofern Sie den/die ursprünglichen Autor(en) und die Quelle ordnungsgemäß nennen, einen Link zur Creative Commons Lizenz beifügen und angeben, ob Änderungen vorgenommen wurden.

Die in diesem Artikel enthaltenen Bilder und sonstiges Drittmaterial unterliegen ebenfalls der genannten Creative Commons Lizenz, sofern sich aus der Abbildungslegende nichts anderes ergibt. Sofern das betreffende Material nicht unter der genannten Creative Commons Lizenz steht und die betreffende Handlung nicht nach gesetzlichen Vorschriften erlaubt ist, ist für die oben aufgeführten Weiterverwendungen des Materials die Einwilligung des jeweiligen Rechteinhabers einzuholen.

Weitere Details zur Lizenz entnehmen Sie bitte der Lizenzinformation auf http://creativecommons.org/licenses/by/4.0/deed.de.

\section{Literatur}

Anger, J., \& Schober, V. (2014). Rechtliche Rahmenbedingungen von Supervision und Coaching in Europa. Wien: ANSE.

Bachmann, T., \& Fietze, B. (2018). Die Digitalisierung von Coaching - Gedanken aus der Perspektive teilnehmender Beobachtung. Organisationsberatung - Supervision - Coaching, 25(3), 231-253. https://doi.org/10.1007/s11613-018-0561-5.

Baumann, M. (2016). Den Nutzen von Coach-Pools in Unternehmen verbessern. In R. Wegener, M. Loebbert \& A. Fritze (Hrsg.), Zur Differenzierung von Handlungsfeldern im Coaching. Die Etablierung neuer Praxisfelder (S. 421-428). Wiesbaden: Springer VS. https://doi.org/10.1007/978-3-658-12140-2_33.

Behrendt, P., \& Greif, S. (2018). Erfolgsfaktoren im Coachingprozess. In S. Greif, H. Möller \& W. Scholl (Hrsg.), Schlüsselkonzepte im Coaching (S. 163-172). Berlin: Springer. https://doi.org/10.1007/ 978-3-662-49483-7_81.

Bühner, M. (2010). Objektivität von Verhaltensbeobachtungen. In K. Westhoff, C. Hagemeister, M. Kersting, F. Lang, H. Moosbrugger, G. Reimann \& G. Stemmler (Hrsg.), Grundwissen für die berufsbezogene Eignungsbeurteilung nach DIN 33430 (S. 78-83). Lengerich: Pabst Science Publishers.

DBVC (2013). Zusammenfassung: Coaching-Marktanalyse 2013. Ergebnisse der 3. Marburger Coaching-Studie 2013. Osnabrück: DBVC.

Diagnostik- und Testkuratorium (2018). Personalauswahl kompetent gestalten. Grundlagen und Praxis der Eignungsdiagnostik nach DIN 33430. Berlin: Springer.

DIN (Hrsg.). (2014). Anforderungen an berufsbezogene Eignungsdiagnostik (DIN 33430:2014). Berlin: Beuth.

Finke, S., \& Kanning, U. P. (2019). Wie wählen Unternehmen ihre Coaches aus? Eine wissenschaftliche Studie gibt Aufschluss. Coaching-Magazin, 12(1), 50-54.

Flanagan, J.C. (1954). The critical incident technique. Psychological Bulletin, 51(4), 327-358. https://doi.org/10.1037/h0061470.

Greif, S. (2015). Evaluation von Coaching als schwer bewertbare Dienstleistung. In A. Schreyögg \& C. Schmidt-Lellek (Hrsg.), Die Professionalisierung von Coaching (S. 47-69). Wiesbaden: Springer VS. https://doi.org/10.1007/978-3-658-08172-0_3.

Gross, P.P., \& Stephan, M. (2011). Coaching aus wirtschaftswissenschaftlicher Sicht - Ergebnisse der Marburger Coaching Studie 2009. In M. Stephan \& P. P. Gross (Hrsg.), Organisation und Marketing von Coaching (S. 3-34). Wiesbaden: Springer VS.

Gross, P.P., \& Stephan, M. (2015). Der Coaching-Markt. Coaching Theorie \& Praxis, 1(1), 15-24. https://doi.org/10.1365/s40896015-0002-9.

De Haan, E., Grant, A.M., Burger, Y., \& Eriksson, P.-O. (2016). A large-scale study of executive and workplace coaching: the re- 
lative contributions of relationship, personality match, and selfefficacy. Consulting Psychology Journal: Practice and Research, 68(3), 189-207. https://doi.org/10.1037/cpb0000058.

Henle, R. (2009). Auswahlprozess für externe Coaches: eine explorative Untersuchung in Großunternehmen. Saarbrücken: VDM.

Heß, T., \& Roth, W.L. (2001). Professionelles Coaching. Eine Expertenbefragung zur Qualitätseinschätzung und -entwicklung. Heidelberg: Asanger.

Kanning, U.P., \& Fricke, P. (2013). Führungserfahrung - Wie nützlich ist sie wirklich? Personalführung, 46(1), 48-53.

Kaul, C. (2005). Coaching bei Volkswagen. Wirtschaftspsychologie aktuell, 12(1), 29-32.

Kaul, C. (2008). Aufbau eines Coaching-Pools - worauf Sie unbedingt achten sollten. Coaching-Magazin, 1(3), 23-26.

Kaul, C., \& Webers, T. (2009). „Wird mein Arbeitsleben zu einem wirklich gelungenen Leben?" Interview mit Dr. Christine Kaul. Coaching-Magazin, 2(2), 10-15.

Kersting, M. (2009). DIN 33430: Akzeptanz durch Qualität, Initiative und Geduld. Zeitschrift für Personalpsychologie, 8(3), 154-158. https://doi.org/10.1026/1617-6391.8.3.154.

Kersting, M. (2010). Qualitätssichernde und qualitätsoptimierende Maßnahmen. In K. Westhoff, C. Hagemeister, M. Kersting, F. Lang, H. Moosbrugger, G. Reimann \& G. Stemmler (Hrsg.), Grundwissen für die berufsbezogene Eignungsbeurteilung nach DIN 33430 (S. 220-231). Lengerich: Pabst Science Publishers.

Klehe, U.-C. (2008). Die DIN 33430 - eine komplexe Norm für eine komplexe Welt. Zeitschrift für Personalpsychologie, 7(4), 183-188. https://doi.org/10.1026/1617-6391.7.4.183.

Kühl, S. (2006). Coaching zwischen Qualitätsproblemen und Professionalisierungsbemühungen. Organisationsberatung - Supervision - Coaching, 13(1), 86-96. https://doi.org/10.1007/s11613006-0008-2.

Kühl, S. (2008a). Die nur fast gelingende Schließung des Personalentwicklungszyklus. Organisationsberatung - Supervision Coaching, 15(2), 137-155. https://doi.org/10.1007/s11613-0080075-7.

Kühl, S. (2008b). Vom Evaluationsdilemma - oder des Kaisers neuen Kleidern. Coaching-Magazin, 1(2), 36.

Kühl, S. (2008c). Die Professionalisierung der Professionalisierer? Das Scharlatanerieproblem im Coaching und der Supervision und die Konflikte um die Professionsbildung. Organisationsberatung Supervision - Coaching, 15(3), 260-294. https://doi.org/10.1007/ s11613-008-0085-5.

Mayring, P. (2015). Qualitative Inhaltsanalyse. Grundlagen und Techniken (12. Aufl.). Weinheim: Beltz.

Müller, O. (2012). Coach-Auswahl im Personalmanagement. Berlin: Cornelsen.

Müller, C., Dollinger, A., \& Limpächer, S. (2015). Internes Coaching: Perspektiven und Trends aus der Praxis. In A. Dollinger \& S. Limpächer (Hrsg.), Internes Coaching. Praxisberichte, Prozesse, Methoden (S. 32-54). Weinheim: Beltz.

Nyuli, E. (2016). Wer darf im Coaching-Pool mitschwimmen? Ein Coach appelliert an die Forschung - Ein Praxisbericht. In R. Wegener, M. Loebbert \& A. Fritze (Hrsg.), Zur Differenzierung von Handlungsfeldern im Coaching (S. 413-420). Wiesbaden: Springer. https://doi.org/10.1007/978-3-658-12140-2_32.

Oellerich, K., Möller, H., \& Freynik, V. (2013). Reine Vertrauenssache? Vertrauen - ein Vorschuss, der gegeben wird, ohne die Konsequenzen genau zu kennen? Coaching-Magazin, 6(4), 47-51.

Rauen, C., \& Steinke, I. (2019). Kompetenz-Anforderungsprofil für Coaches. In DBVC (Hrsg.), Leitlinien und Empfehlungen für die Entwicklung von Coaching als Profession. Kompendium mit den Professionsstandards des DBVC (Bd. 5, S. 42-51). Osnabrück: Deutscher Bundesverband Coaching.
Reimann, G. (2010). Arbeits- und Anforderungsanalyse. In K. Westhoff, C. Hagemeister, M. Kersting, F. Lang, H. Moosbrugger, G. Reimann \& G. Stemmler (Hrsg.), Grundwissen für die berufsbezogene Eignungsbeurteilung nach DIN 33430 (S. 102-116). Lengerich: Pabst Science Publishers.

Reimann, G., Frenzel, T., Michalke, S., \& Peper, M. (2009a). Verbreitung und Akzeptanz der DIN 33430 - Eine zweite Stellungnahme. Zeitschrift für Personalpsychologie, 8(1), 35-39. https://doi. org/10.1026/1617-6391.8.1.35.

Reimann, G., Frenzel, T., Michalke, S., \& Peper, M. (2009b). DIN 33430 - Quo vadis? Zeitschrift für Personalpsychologie, 8(3), 156-158. https://doi.org/10.1026/1617-6391.8.3.156.

Reimer, H. (2010). Wer soll in unseren Coach-Pool? Professionell Coaches auswählen. Coaching-Magazin, 3(1), 28-32.

Reimer, H., \& Fengel, K. (2010). Coach-Assessment bei der RheinEnergie AG. Schritte zum Aufbau eines Coach-Pools. Personalführung, 43(9), 38-42.

Schermuly, C.C., \& Graßmann, C. (2018). A literature review on negative effects of coaching - what we know and what we need to know. Coaching: An International Journal of Theory, Research and Practice, 12(1), 39-66. https://doi.org/10.1080/17521882. 2018.1528621

Schilling, L. (2019). Coaching im Unternehmen - Closed Shops ohne Zugangskontrolle? Untersuchung zum Status Quo der Auswahl von Coaches und Ableitung eines Idealmodells nach DIN 33430 (unveröffentlichte Masterarbeit). Köln: Hochschule Fresenius.

Scholer, \& Lendner (2012). Stadt München: Mühselige Suche nach guten Coachs. wirtschaft + weiterbildung, 24(3), 46-49.

Schoorman, F.D., Mayer, R.C., \& Davis, J.H. (2007). An integrative model of organizational trust: past, present, and future. Academy of Management Review, 32(2), 344-354. https://doi.org/10.5465/ amr.2007.24348410.

Schuler, C.C. (2019). Erfolgreiches Business-Coaching. Positive Wirkungen, unerwünschte Nebenwirkungen und vermeidbare Abbrüche. Weinheim: Beltz.

Schuler, H. (2014). Psychologische Personalauswahl. Bd. 4. Göttingen: Verlag für angewandte Psychologie.

Steinle, C., Eichenberg, T., \& Dietrich, M. (2009). Wie den ,richtigen Coach auswählen? Empfehlungen zur kompetenzbasierten Auswahl von Coachs. Personalführung, 42(3), 60-67.

Stephan, M., \& Gross, P.P. (2011). Coaching aus wirtschaftswissenschaftlicher Sicht - Ergebnisse der Marburger Coaching Studie 2009. In M. Stephan \& P.-P. Gross (Hrsg.), Organisation und Marketing von Coaching (S. 3-34). Wiesbaden: Springer VS.

Stephan, M., \& Rötz, C. (2018). Coaching-Marktanalyse 2016/17. Ergebnisse der 4. Marburger Coaching Studie 2016/17 (Discussion Papers on Strategy and Innovation 18-01). Marburg: PhilippsUniversity Marburg, Department of Technology and Innovation Management. https://www.uni-marburg.de/de/fb02/professuren/ bwl/bwl01/forschung/2018-01_coaching_onelineversion.pdf. Zugegriffen: 4. Nov. 2019.

Stiftung Warentest (Hrsg.). (2014). Den richtigen Coach finden: Nicht nur die Chemie muss stimmen. Berlin: Stiftung Warentest. www.test.de/Den-richtigen-Coach-finden-Nicht-nur-dieChemie-muss-stimmen-4697530-0/. Zugegriffen: 29. August 2019.

Tonhäuser, C. (2012). Coaching als Instrument der Personalentwicklung: Ergebnisse einer empirischen Studie. Vortrag auf dem 2. Internationalen Coaching-Forschungskongress „Coaching meets Research“, Basel, 6. Juni 2012.

Webers, T. (2013). Coaching-Kompetenzen: Behauptungen und Kritik. Organisationsberatung - Supervision - Coaching, 20(4), 479-487. https://doi.org/10.1007/s11613-013-0351-z.

Webers, T. (2015). Systemisches Coaching. Wiesbaden: Springer. 
Weinert, S., \& Stulle, K. (2015). Executive Assessment. Instrumente, Trends, Herausforderungen. Wiesbaden: Springer Gabler.

Westhoff, K., Hagemeister, C., Kersting, M., Lang, F., Moosbrugger, H., Reimann, G., \& Stemmler, G. (Hrsg.). (2010). Grundwissen für die berufsbezogene Eignungsbeurteilung nach DIN 33430. Lengerich: Pabst Science Publishers.

Publisher's Note Springer Nature remains neutral with regard to jurisdictional claims in published maps and institutional affiliations. 